\title{
REVIEW
}

\section{Optical manipulation from the microscale to the nanoscale: fundamentals, advances and prospects}

\author{
Dongliang Gao ${ }^{1,2}$, Weiqiang Ding ${ }^{3}$, Manuel Nieto-Vesperinas ${ }^{4}$, Xumin Ding ${ }^{3}$, Mahdy Rahman ${ }^{1,5}$, \\ Tianhang Zhang ${ }^{1,6}$, ChweeTeck Lim ${ }^{6,7}$ and Cheng-Wei Qiu ${ }^{1,6,8}$
}

Since the invention of optical tweezers, optical manipulation has advanced significantly in scientific areas such as atomic physics, optics and biological science. Especially in the past decade, numerous optical beams and nanoscale devices have been proposed to mechanically act on nanoparticles in increasingly precise, stable and flexible ways. Both the linear and angular momenta of light can be exploited to produce optical tractor beams, tweezers and optical torque from the microscale to the nanoscale. Research on optical forces helps to reveal the nature of light-matter interactions and to resolve the fundamental aspects, which require an appropriate description of momenta and the forces on objects in matter. In this review, starting from basic theories and computational approaches, we highlight the latest optical trapping configurations and their applications in bioscience, as well as recent advances down to the nanoscale. Finally, we discuss the future prospects of nanomanipulation, which has considerable potential applications in a variety of scientific fields and everyday life.

Light: Science \& Applications (2017) 6, e17039; doi:10.1038/Isa.2017.39; published online 22 September 2017

Keywords: biochemical manipulation; microscale; nanoscale; optical force; optical tweezer; plasmonics

\section{INTRODUCTION}

Optical manipulation (OM), such as optical cooling ${ }^{1}$, trapping ${ }^{2}$, binding $^{3-5}$, sorting and transporting ${ }^{6,7}$ by utilizing optical forces, has experienced intensive development in the past 40 years. $\mathrm{OM}$ is currently one of the most important tools in many scientific areas, including optics ${ }^{8}$, atomic physics ${ }^{9,10}$, biological science ${ }^{11}$ and chemistry ${ }^{12}$. Recently, with the rapid progression of nanotechnology, novel features and trends of $\mathrm{OM}$ have made great progress at subwavelength and nanometer scales.

In traditional $\mathrm{OM}$ studies, the configuration of a dielectric or metallic sphere in the focus of a light beam is usually investigated by considering the scattering of its fundamental mode, which is often Gaussian. Recently, however, OM has gone beyond this scenario, and complex configurations have been investigated. For example, OM resolution has been increased beyond the Rayleigh limit by extending it to the near-field, thus taking advantage of non-radiative optical waves $^{13}$. The manipulated object can be magneto-dielectric ${ }^{14}$, chiral ${ }^{15}$ or multilayer coated ${ }^{16,17}$ rather than a single dielectric body. In addition, the object may be asymmetric, such as a rotator or gearwheel. Meanwhile, the incident light is usually composed of special wavefields, such as non-diffraction Bessel beams stemming from multiple beam interference, or more complex light fields generated by a spatial light modulator. A representative example is the recently proposed optical tractor beams ${ }^{18-24}$, which can exert a negative radiation pressure, or non-conservative force, on a body, thus pulling it towards the light source ${ }^{25}$.

In addition, when objects are resonant with the incident light ${ }^{26}$, the optical force may be greatly enhanced. In this way, when a single structure simultaneously supports both optical and mechanical resonances, novel phenomena can be expected and achieved ${ }^{27}$. Photonic forces on nanoparticles have recently been related to the fundamental van der Waals and Casimir interactions ${ }^{28-32}$, and it has been shown how stochastic photonic forces can be created and tailored by appropriately designing and controlling the coherence and statistical properties of fluctuating optical sources ${ }^{33}$. Using $\mathrm{OM}$ as a powerful tool, many new applications have been demonstrated in biochemical research for biological cell constituents, DNA and biopolymers $^{34-36}$.

Although several excellent articles have reviewed various aspects of $\mathrm{OM}^{37-43}$, this tool has rapidly developed in diverse domains, especially during the past few years. New achievements have emerged and have affected various scientific fields. In this review, we attempt to provide a full picture of the latest advances in $\mathrm{OM}$ as well as insights and perspectives for future applications, ranging from the now century-old

\footnotetext{
${ }^{1}$ Department of Electrical and Computer Engineering, National University of Singapore, Singapore 117583, Singapore; ${ }^{2}$ College of Physics, Optoelectronics and Energy, Soochow University, Suzhou 215006, China; ${ }^{3}$ Department of Physics, Harbin Institute of Technology, Harbin 150001, China; ${ }^{4}$ Instituto de Ciencia de Materiales de Madrid, Consejo Superior de Investigaciones Científicas, Campus de Cantoblanco, Madrid 28049, Spain; ${ }^{5}$ Department of Electrical and Computer Engineering, North South University, Dhaka 1229, Bangladesh; ${ }^{6}$ NUS Graduate School for Integrative Sciences and Engineering, National University of Singapore, Singapore 117456 , Singapore; ${ }^{7}$ Department of Mechanical Engineering, National University of Singapore, Singapore 117576, Singapore and ${ }^{8}$ SZU-NUS Collaborative Innovation Center for Optoelectronic Science and Technology, Shenzhen University, Shenzhen 518060, China

Correspondence: CW Quu, Email: eleqc@nus.edu.sg

Received 18 October 2016; revised 28 February 2017; accepted 7 March 2017; accepted article preview online 9 March 2017
} 
fundamental dilemma of photon momentum transfer in media to applications on micrometer-sized or smaller objects and structures, which have recently attracted considerable attention. We start with the fundamental computations of optical force and then emphasize the recently developed OM tractor beams, plasmonic nanotweezers and biochemical applications. First, we present a detailed description of the physical origins, nature and determinations of optical forces, which are useful to probe the underlying physical aspects of momentum transfer. Thereafter, we review the OM of structured beams based on optical pulling, optical rotation and optical binding. Then, approaches to enhance the optical force on nanoscale particles by plasmonics are presented, and applications of OM in life sciences, such as in biological cells, DNA and other types of biopolymers, are discussed. Finally, our perspective of future OM developments and potential applications is presented.

\section{FUNDAMENTALS OF OPTICAL FORCES AND COMPUTATIONAL APPROACHES}

We start this section with a discussion of the nature of the optical force on particles, that is, the physical origin and calculation approaches. Generally, this force is a consequence of a change in momentum carried by photons $s^{1,2,44}$. When a particle is micrometersized or smaller, the dominant mechanical actions are radiation pressure and the Lorentz force, not gravitational forces. In 1619, Kepler first suggested that the pressure of light causes comet tails to always point away from the Sun ${ }^{45,46}$. This theory was extended by Maxwell and was confirmed experimentally by Nicolas and Hull ${ }^{45,46}$. However, the concept that electromagnetic fields or photons carry momentum led to several problems. For example, the magnitude of radiation pressure is conveniently expressed in the form of momentum carried by a single photon ${ }^{46}$. With this concept well understood in free space $e^{46}$, researchers naturally considered its counterpart in matter ${ }^{46,47}$, especially inside a body ${ }^{47}$, because almost all OM schemes address objects suspended in a fluid or linked to a solid. The ensuing debate on photon momentum $\boldsymbol{p}$ in matter, known as the AbrahamMinkowski and related controversies in electrodynamics, is whether one should write momentum as

$$
p_{\mathrm{Min}}=\hbar k=\frac{n \hbar \omega}{c} \text { or } p_{\mathrm{Abr}}=m v=\frac{\hbar \omega}{n c}
$$

where $\hbar$ is the reduced Planck's constant, $\omega$ represents the angular frequency of light, $c$ is the speed of light in vacuum and $n$ denotes the refractive index of the background medium. A possible resolution of this problem regarding photon momentum was proposed by Barnett ${ }^{48}$, who argues that $p_{\mathrm{Min}}$ and $p_{\mathrm{Abr}}$ describe two entirely different concepts. Total momentum is always a single conserved quantity, where Minkowski's photon momentum is the canonical momentum of a photon and Abraham's photon momentum is the kinetic momentum of a photon ${ }^{48}$.

Consequently, the calculation of the total optical force on a body embedded in a medium has different versions, which can be derived by integrating the momentum flux over any closed surface $\sigma$ surrounding this object. The time-average force on a particle of any size is ${ }^{49,50}$

$$
\boldsymbol{F}=\int_{\sigma}\langle\hat{T}\rangle \cdot \boldsymbol{n} \mathrm{d} s
$$

where $\hat{T}$ is the Maxwell stress tensor, $\boldsymbol{n}$ is the outward normal to the surface $\sigma$ and $\langle\cdot\rangle$ represents the time-average operation. Initially, Minkowski ${ }^{51,52}$ suggested that a field energy-momentum tensor should preserve its form in all Lorentz frames. According to him, the Maxwell stress tensor should be

$$
\hat{T}_{\mathrm{M}}=\boldsymbol{D} \boldsymbol{E}+\boldsymbol{B} \boldsymbol{H}-\frac{1}{2}(\boldsymbol{B} \cdot \boldsymbol{H}+\boldsymbol{D} \cdot \boldsymbol{E}) \hat{I}
$$

where $\hat{I}$ is the unit tensor. This stress tensor was derived from the optical momentum transfer from the background to the embedded body. Several experiments have supported Minkowski's theory. Experimental data in favor of the Minkowski formulation are found in detail in Refs 48,53,54.

However, Abraham suggested that the electromagnetic stress tensor should be symmetric ${ }^{55-57}$ :

$$
\hat{T}_{\mathrm{A}}=\frac{1}{2}[\boldsymbol{D E}+\boldsymbol{E D}+\boldsymbol{B} \boldsymbol{H}+\boldsymbol{H B}-(\boldsymbol{B} \cdot \boldsymbol{H}+\boldsymbol{D} \cdot \boldsymbol{E}) \hat{I}]
$$

Experiments supporting the Abraham formulation can be found in detail in Refs 45,58-60. The controversies in the area of optical force calculation can be resolved if the corresponding ranges of validity of the optical force formulations are clearly identified ${ }^{14,61-63}$. However, in most configurations addressing time-averaged forces, as reviewed in this paper, both theories yield the same time-averaged optical force ${ }^{54}$. In fact, the difference between Minkowski's force and Abraham's force arises mainly in the time domain ${ }^{54}$. There are approaches other than Equation (2), for example, using different force density methods ${ }^{64}$. However, in several experiments where in the background was considered as a material medium rather than air or vacuum, the different volumetric force formulations predicted distinct magnitudes of the time-averaged force on an embedded scatterer ${ }^{63}$. Therefore, the formulations of Minkowski and Abraham are considered the most reliable approaches for the time-averaged force on objects of any size or shape $\mathrm{e}^{54}$.

Early in the 1970s, optical forces from focused laser beams, which could accelerate and trap particles at the micron scale, were observed in experiments ${ }^{2}$. There are two basic components of force in optical tweezers: gradient force and scattering force. The gradient force comes from the intensity gradient of the electric field of the focused beam, and the scattering force is caused by the optical momentum of the light transferred to the particle. The conservative gradient force points to the position of the electric intensity maxima, whereas the nonconservative scattering force from beams pushes the object along the propagation direction. The balance between these two forces produces an equilibrium point near the focus. Optical tweezers, based on field gradients, rely on changing this equilibrium point to manipulate trapped bodies in three dimensions. Optical tweezers have multiple applications in biological and physical sciences.

In general, an illuminated dipolar magneto-dielectric particle can develop electric and magnetic dipole moments in response to an electromagnetic field ${ }^{65}$. Hence, the resulting optical forces can be regarded as the sum of forces exerted on the induced dipoles and the interaction between the electric and magnetic dipoles. Compared with the method that integrates the Maxwell stress tensor on the surface of objects, this decomposition helps us to understand the different contributions of an external electromagnetic field to the optical force. In the following paragraphs, we consider a magneto-dielectric nanoparticle with constitutive parameters $\varepsilon_{\mathrm{p}}$ and $\mu_{\mathrm{p}}$, embedded in a non-absorbing medium with permittivity $\varepsilon$, permeability $\mu$ and refractive index $n=\sqrt{\varepsilon \mu}$. This object is illuminated by an incident field with electric and magnetic vectors $\boldsymbol{E}_{\mathrm{i}}$ and $\boldsymbol{B}_{\mathrm{i}}$. A harmonic time dependence $\exp (-i \omega t)$ is assumed throughout. The time-averaged total electromagnetic force on the particle is given by the Minkowski 
stress tensor ${ }^{50}$

$$
\begin{aligned}
\langle\boldsymbol{F}\rangle= & \frac{1}{2} \operatorname{Re}\left(\int _ { S } \left[\varepsilon(\boldsymbol{E} \cdot \boldsymbol{n}) \boldsymbol{E}^{*}+\mu^{-1}(\boldsymbol{B} \cdot \boldsymbol{n}) \boldsymbol{B}^{*}-\frac{1}{2}\left(\varepsilon|\boldsymbol{E}|^{2}\right.\right.\right. \\
& \left.\left.\left.+\mu^{-1}|\boldsymbol{B}|^{2}\right) \boldsymbol{n}\right] \mathrm{d} S\right)
\end{aligned}
$$

where the asterisk denotes a complex conjugate, $S$ is any surface that encloses the particle and $\boldsymbol{n}$ is its local unit outward normal. The electric and magnetic fields in Equation (5) are total fields, that is, $\boldsymbol{E}_{\mathrm{i}}+\boldsymbol{E}^{(\mathrm{s})}$ and $\boldsymbol{B}_{\mathrm{i}}+\boldsymbol{B}^{(\mathrm{s})}$. For the flow evaluation of the outside surface enclosing an object and assuming the embedding background is a linear homogeneous isotropic medium, the Minkowski and Abraham stress tensors coincide.

If the object is considered as dipolar in the wide sense (the particle size is much smaller than $\lambda / n$, where $\lambda$ is the incident wavelength in the background medium), its electric and magnetic polarizabilities $\alpha_{\mathrm{e}}$ and $\alpha_{\mathrm{m}}$ can be written in terms of the coefficients $a_{1}$ and $b_{1}$ of the first Mie electric and magnetic partial waves as follows: $\alpha_{\mathrm{e}}=i \frac{3 \varepsilon}{2 k^{3}} a_{1}, \alpha_{\mathrm{m}}=$ $i \frac{3}{2 \mu k^{3}} b_{1}$ and $k=n \omega / c$. Then, the $i$ th Cartesian component of the averaged force may be expressed in the following form: ${ }^{14,66}$

$$
\begin{aligned}
\left\langle\boldsymbol{F}_{i}\right\rangle= & \frac{1}{2} \operatorname{Re}\left(\boldsymbol{p}_{j} \partial_{i} \boldsymbol{E}_{j}^{*}+\boldsymbol{m}_{j} \partial_{i} \boldsymbol{B}_{j}^{*}\right) \\
& -\frac{k^{4}}{3} \sqrt{\frac{\mu}{\varepsilon}} \operatorname{Re}\left(\left(\boldsymbol{p} \times \boldsymbol{m}^{*}\right)_{i}\right) \quad(i, j=1,2,3)
\end{aligned}
$$

where $\boldsymbol{p}$ and $\boldsymbol{m}$ denote the electric and magnetic dipole moments of the particle induced by the incident field, $\boldsymbol{p}=\alpha_{\mathrm{e}} \boldsymbol{E}^{(i)}$ and $\boldsymbol{m}=\alpha_{\mathrm{m}} \boldsymbol{B}^{(i)}$, respectively. In Equation (6), Einstein's summation convention is understood over all repeated indices. The three terms of Equation (6) represent the forces due to the induced dipoles: electric $\left\langle F_{\mathrm{e}}\right\rangle$, magnetic $\left\langle\boldsymbol{F}_{\mathrm{m}}\right\rangle$ and interference $\left\langle\boldsymbol{F}_{\mathrm{e}-\mathrm{m}}\right\rangle$ between them. The interference component is associated with the asymmetry of the particle's differential scattering cross-section or angular distribution of the scattered intensity under a plane wave or Gaussian beam illumination. This effect in turn is related to interesting scattering effects, such as the emission of zero-backward (when $\varepsilon^{-1} \alpha_{\mathrm{e}}=\mu \alpha_{\mathrm{m}}$ ) or almost zero-forward scattered intensity (when $\operatorname{Re}\left(\varepsilon^{-1} \alpha_{\mathrm{e}}\right)=-\operatorname{Re}\left(\mu \alpha_{\mathrm{m}}\right)$ and $\left.\operatorname{Im}\left(\varepsilon^{-1} \alpha_{\mathrm{e}}\right)=\operatorname{Im}\left(\mu \alpha_{\mathrm{m}}\right)\right)^{67}$, known as the first and second Kerker conditions, respectively ${ }^{68-71}$.

The three components $\left\langle\boldsymbol{F}_{\mathrm{e}}\right\rangle,\left\langle\boldsymbol{F}_{\mathrm{m}}\right\rangle$ and $\left\langle\boldsymbol{F}_{\mathrm{e}-\mathrm{m}}\right\rangle$ of Equation (6) for a general magneto-dielectric dipolar particle may also be written in more explicit form as follows ${ }^{14}$ :

$$
\begin{array}{r}
\left\langle\boldsymbol{F}_{\mathrm{e}}\right\rangle=\frac{1}{\varepsilon} \operatorname{Re}\left(\alpha_{e}\right) \nabla\left\langle\boldsymbol{U}_{\mathrm{e}}\right\rangle+\mu_{c}^{-} \operatorname{Im}\left(\alpha_{e}\right)\langle\boldsymbol{S}\rangle-\frac{\omega}{\varepsilon} \operatorname{Im}\left(\alpha_{e}\right) \nabla \times\left\langle\boldsymbol{L}_{\mathrm{Se}}\right\rangle \\
\left\langle\boldsymbol{F}_{\mathrm{m}}\right\rangle=\frac{1}{\varepsilon} \operatorname{Re}\left(\alpha_{\mathrm{m}}\right) \nabla\left\langle\boldsymbol{U}_{\mathrm{m}}\right\rangle+\mu_{c}^{k} \operatorname{Im}\left(\alpha_{\mathrm{m}}\right)\langle\boldsymbol{S}\rangle-\frac{\omega}{\varepsilon} \operatorname{Im}\left(\alpha_{\mathrm{m}}\right) \nabla \times\left\langle\boldsymbol{L}_{\mathrm{Sm}}\right\rangle \\
\left\langle\boldsymbol{F}_{\mathrm{e}-\mathrm{m}}\right\rangle=-\frac{k^{4}}{3} \sqrt{\frac{\mu}{\varepsilon}} \operatorname{Re}\left(\alpha_{\mathrm{e}} \alpha_{\mathrm{m}}^{*}\right) \operatorname{Re}\left(\boldsymbol{E} \times \boldsymbol{B}^{*}\right) \\
+\frac{k^{4}}{3} \mu \operatorname{Im}\left(\alpha_{\mathrm{e}} \alpha_{\mathrm{m}}^{*}\right)\left[\frac{1}{2} \nabla|\boldsymbol{E}|^{2}-\operatorname{Re}\left(\left(\boldsymbol{E}^{*} \cdot \nabla\right) \boldsymbol{E}\right)\right]
\end{array}
$$

where $\left\langle\boldsymbol{U}_{\mathrm{e}, \mathrm{m}}\right\rangle$ is the time-average of the electric (magnetic) energy density, $\left\langle\boldsymbol{L}_{\mathrm{Se}, \mathrm{Sm}}\right\rangle$ is the electric (magnetic) spin density of the optical field and $\langle S\rangle$ stands for the Poynting vector ${ }^{14}$

$$
\begin{aligned}
& \langle\boldsymbol{S}\rangle=\frac{1}{2 \mu} \operatorname{Re}\left(\boldsymbol{E} \times \boldsymbol{B}^{*}\right), \quad\left\langle\boldsymbol{U}_{\mathrm{e}}\right\rangle=\frac{1}{4} \varepsilon|\boldsymbol{E}|^{2}, \quad\left\langle\boldsymbol{U}_{\mathrm{m}}\right\rangle=\frac{1}{4 \mu}|\boldsymbol{B}|^{2} \\
& \left\langle\boldsymbol{L}_{\mathrm{Se}}\right\rangle=\frac{\varepsilon}{4 \omega i} \operatorname{Im}\left(\boldsymbol{E}^{*} \times \boldsymbol{E}\right), \quad\left\langle\boldsymbol{L}_{\mathrm{Sm}}\right\rangle=\frac{1}{4 \mu \omega i} \operatorname{Im}\left(\boldsymbol{B}^{*} \times \boldsymbol{B}\right)
\end{aligned}
$$

The first terms of Equations (7) and (8) are the gradient components that are responsible for electric and magnetic optical tweezers. The remaining terms constitute non-conservative electric and magnetic forces $\left\langle\boldsymbol{F}_{\mathrm{e}}^{\mathrm{nc}}\right\rangle$ and $\left\langle\boldsymbol{F}_{\mathrm{m}}^{\mathrm{nc}}\right\rangle$. The second term describes the radiation pressure or scattering component expressed by the time-averaged energy flow. This term is the only contributing one if the incident wave field reduces to a plane wave. The third term accounts for the curl force due to $\operatorname{spin}^{72,73}$.

The time-average Poynting vector can be decomposed into the sum of time-averages of the electric orbital momentum density $\left\langle\boldsymbol{P}_{\mathrm{e}}^{\mathrm{O}}\right\rangle$ and spin momentum density $\left\langle\boldsymbol{P}_{\mathrm{e}}^{S}\right\rangle^{74}$ or alternatively as the sum of the magnetic orbital momentum density $\left\langle\boldsymbol{P}_{\mathrm{m}}^{\mathrm{O}}\right\rangle$ and spin momentum density $\left\langle\boldsymbol{P}_{\mathrm{m}}^{\mathrm{S}}\right\rangle$ as follows:

$$
\begin{aligned}
\langle\boldsymbol{S}\rangle_{i} & =\frac{1}{2 \omega \mu}\left\{\operatorname{Im}\left(\boldsymbol{E}_{j}^{*} \partial_{i} \boldsymbol{E}_{j}\right)+\frac{1}{2} \nabla \times \operatorname{Im}\left(\left(\boldsymbol{E}^{*} \times \boldsymbol{E}\right)_{i}\right\}\right. \\
& =\left\langle\boldsymbol{P}_{\mathrm{e}}^{\mathrm{O}}\right\rangle+\left\langle\boldsymbol{P}_{\mathrm{e}}^{\mathrm{S}}\right\rangle, \quad(i, j=1,2,3) \\
\langle\boldsymbol{S}\rangle_{i} & =\frac{1}{2 \omega \varepsilon \mu^{2}}\left\{\operatorname{Im}\left(\boldsymbol{B}_{j}^{*} \partial_{i} \boldsymbol{B}_{j}\right)+\frac{1}{2} \nabla \times \operatorname{Im}\left(\boldsymbol{B}^{*} \times \boldsymbol{B}\right)_{i}\right\} \\
& =\left\langle\boldsymbol{P}_{\mathrm{m}}^{\mathrm{O}}\right\rangle+\left\langle\boldsymbol{P}_{\mathrm{m}}^{\mathrm{S}}\right\rangle, \quad(i, j=1,2,3)
\end{aligned}
$$

From Equations (7),(8) (11) and (12), one can identify the nonconservative component of the forces from the electric and magnetic dipoles as proportional to the respective electric and magnetic orbital momentum densities, that is, $\left\langle\boldsymbol{F}_{\mathrm{e}}^{\mathrm{nc}}\right\rangle=\omega \mu \operatorname{Im}\left(\alpha_{\mathrm{e}}\right)\left\langle\boldsymbol{P}_{\mathrm{e}}^{\mathrm{O}}\right\rangle ;\left\langle\boldsymbol{F}_{\mathrm{m}}^{\mathrm{nc}}\right\rangle=$ $\omega \varepsilon \mu^{2} \operatorname{Im}\left(\alpha_{\mathrm{m}}\right)\left\langle\boldsymbol{P}_{\mathrm{m}}^{\mathrm{O}}\right\rangle$. Alternatively, the electric and magnetic nonconservative optical forces can be represented in terms of the classical radiation pressure expressed by the linear momentum density or Poynting vector $\langle\boldsymbol{S}\rangle$ that characterizes the energy flow plus the curl of the spin density. For the latter case, the introduction of the linear momentum $\langle\boldsymbol{S}\rangle$ automatically arises in the non-conservative forces due to the appearance of the electric or magnetic spin momentum densities $\left\langle\boldsymbol{P}_{\mathrm{e}, \mathrm{m}}^{\mathrm{S}}\right\rangle=\nabla \times\left\langle\boldsymbol{L}_{\mathrm{Se}, \mathrm{Sm}}\right\rangle$. Whereas the action of the orbital momentum is physically well understood, the role of the spin momentum is a subject of active study. Equations (11) and (12) show the interference force $\left\langle\boldsymbol{F}_{\mathrm{e}-\mathrm{m}}\right\rangle$, given by the imaginary part of $\boldsymbol{E} \times \boldsymbol{B}^{\star}$, which characterizes the 'reactive' or 'stored energy' and its alternating flow ${ }^{50}$. These two equations show that in addition to the linear momentum $\langle\boldsymbol{S}\rangle,\left\langle\boldsymbol{F}_{\mathrm{e}, \mathrm{m}}^{\mathrm{nc}}\right\rangle$ contains a new momentum $\operatorname{Re}\left(\boldsymbol{E}^{\star} \cdot \nabla\right) \boldsymbol{E}$ whose significance is still being studied ${ }^{75}$.

Magneto-dielectric particles were previously considered hypothetical and difficult to obtain in nature. On the basis of a magneto-dielectric material, unusual zero backscattering or minimum forward-scattering effects were predicted at certain wavelengths by Kerker et al. ${ }^{69}$ as a consequence of the interaction between the electric and magnetic dipoles. It is possible to build blocks of metamaterials ${ }^{76}$, scatterers or optical antennas due to their unique emission directionality properties. Recent works have shown that dielectric spheres of relatively high refractive index $n_{\mathrm{p}}$ behave as magneto-dielectric particles and exhibit a remarkably scaling property. According to their scattering crosssection, these magneto-dielectric particles are fully characterized by the excitation of either their electric or magnetic dipoles, associated with the Mie coefficients $a_{1}$ and $b_{1}$, respectively, at constant values of the size parameter $y=n_{\mathrm{p}} k a$. If $n_{\mathrm{p}}$ varies, either $k$ or $a$ may be changed accordingly, leaving the value of size parameter at which those excitations appear unchanged. Such dipoles may equally appear in semiconductor particles by incident waves in either the visible or nearinfrared ranges (depending on their sizes) or in ceramics in the microwave range. Such magneto-dielectric nanoparticles, with the corresponding theory of optical forces established as shown above, offer a new twist for $\mathrm{OM}^{77}$. 
As an example, we consider a silicon sphere of radius $a=230 \mathrm{~nm}$ and $\varepsilon_{\mathrm{p}}=3.5$ in a vacuum $(\varepsilon=\mu=1)$ illuminated by a plane wave of unit amplitude. The time-averaged optical force it experiences is purely a non-conservative scattering force ${ }^{14}$,

$$
\langle\boldsymbol{F}\rangle=\frac{k}{2}\left\{\frac{\operatorname{Im}\left(\alpha_{\mathrm{e}}\right)}{\varepsilon}+\mu \operatorname{Im}\left(\alpha_{\mathrm{m}}\right)-\frac{2 k^{3} \mu}{3 \varepsilon}\left(\operatorname{Re}\left(\alpha_{\mathrm{e}}\right) \operatorname{Re}\left(\alpha_{\mathrm{m}}\right)+\operatorname{Im}\left(\alpha_{\mathrm{e}}\right) \operatorname{Re}\left(\alpha_{\mathrm{m}}\right)\right)\right\}
$$

The three terms of Equation (13) correspond to the aforementioned components $\left\langle\boldsymbol{F}_{\mathrm{e}}\right\rangle,\left\langle\boldsymbol{F}_{\mathrm{m}}\right\rangle$ and $\left\langle\boldsymbol{F}_{\mathrm{e}-\mathrm{m}}\right\rangle$, respectively. The magnitudes of the magnetic term $\left\langle\boldsymbol{F}_{\mathrm{m}}\right\rangle$ and the electric-magnetic interference term $<F_{\mathrm{e}-\mathrm{m}}>$ contribution to the time-averaged total forces have peaks in the region of the magnetic dipole resonance, as shown in Figure 1a and $1 \mathrm{~b}$. Under the first Kerker condition (zero backscattering differential crosssection, see the second vertical line in Figure $1 \mathrm{~b}),\left\langle F_{\mathrm{e}}\right\rangle=\left\langle\boldsymbol{F}_{\mathrm{m}}\right\rangle=$ $-\left\langle F_{\mathrm{e}-\mathrm{m}}\right\rangle$, so the resulting force represents a particle with a purely electric or magnetic dipole. For a plane wave, the negative $\left\langle\boldsymbol{F}_{\mathrm{e}-\mathrm{m}}>\right.$ cannot overcome the positive $\left\langle\boldsymbol{F}_{\mathrm{e}}\right\rangle+\left\langle\boldsymbol{F}_{\mathrm{m}}\right\rangle$. However, as shown in the next section, the positive terms of the electric and magnetic components are overcome by the interference term for a Bessel beam, which in this way constitutes a tractor beam.

By contrast, under the second Kerker condition (minimum forward-scattering differential cross-section, see the first vertical line in Figure 1b), the three force components reinforce each other because they are all positive. There are also regions of the spectrum, $\operatorname{Re}\left(\alpha_{\mathrm{e}}\right)$ $<<\operatorname{Im}\left(\alpha_{\mathrm{e}}\right)$ and $\operatorname{Re}\left(\alpha_{\mathrm{m}}\right)<<\operatorname{Im}\left(\alpha_{\mathrm{m}}\right)$, where the corresponding dipolar scattering force has larger values. These regions have recently been observed in an experiment ${ }^{78}$. This situation is opposite to those of conventional nano-optical tweezers, whereby gradient forces, rather than the radiation pressure, are dominant.

\section{NON-CONSERVATIVE FORCES AND OPTICAL TORQUE}

In the past decade, 'tractor beams' that drag trapped objects towards a light source, have attracted substantial attention ${ }^{19-21,79,80}$. Except for gain materials ${ }^{20,81,82}$, due to momentum conservation, the optical force for paraxial illumination is positive, namely, it pushes the object away from the light source. Recently, however, several exotic light structures have been proposed to trap objects moving towards a light

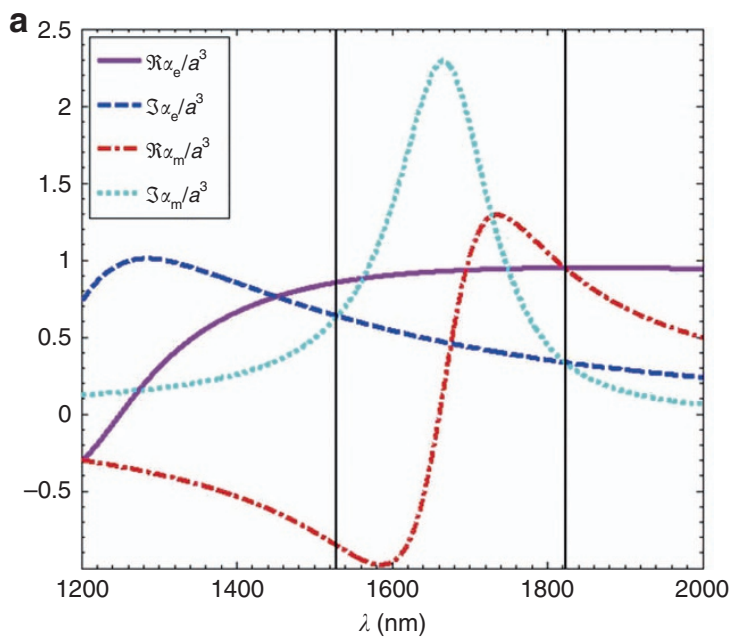

source, such as optical vortex beams ${ }^{83}$, optical solenoid beams ${ }^{84}$, gradientless Bessel beams ${ }^{18,21,22,85,86}$, structured nondiffracting beams ${ }^{19,20}$ and holographic optical beams ${ }^{87-92}$.

It has been demonstrated ${ }^{20}$ that there is a region of negative optical force (NOF) for a class of designed optical beams (Figure 2a). The $\mathrm{NOF}$ is located in the vicinity of a local intensity minimum and acts up to long distances along the propagation direction of the illuminating wave. However, the regions of the NOF are small compared to the incident wavelength. Thus, a nondiffracting beam constituted by 24 plane waves with the same $k_{z}$ component was used ${ }^{19}$. According to the conservation of linear momentum, the polarization and phase of the plane waves were optimized so that the differential scattering crosssection along the propagation direction was maximized. In this case, the total force on a cluster of 160 spheres was opposite to the direction of wave propagation. The structured nondiffracting beam could exert a negative optical force along the extent of the beam for objects of any shape or size. Meanwhile, an optical conveyor belt was built based on the standing wave formed through the interference of two counterpropagating Bessel beams ${ }^{93}$. Controlled delivery of objects over hundreds of micrometers in size was achieved by manipulating the relative phase of both Bessel beams. Alternatively, a negative optical force can be obtained by using a single nonparaxial Bessel beam with small projection of momentum in the light propagation direction ${ }^{18,22,85}$. Suitable particles can be pulled all the way to the light source using nonparaxial Bessel beams, known as 'tractor beams'. Such beams are diffraction-free and can be realized in experiments ${ }^{94}$. Bessel beams also have a self-healing property, whereby the beam wavefront is restored after being scattered by an object, making long distance $\mathrm{OM}$ possible. The diffractionless and self-healing nature of Bessel beams can be utilized to simultaneously trap multiple particles in separated positions with only a single beam ${ }^{95}$. Similar to Bessel beams, the Airy beam is also nondiffracting and self-healing, but it experiences transverse acceleration ${ }^{96}$. It is this unique characteristic that can transport biological objects along a curved trajectory; thus, Airy beams could be used to clear biological cells from a chamber ${ }^{97}$.

For a magneto-dielectric particle of linear size much smaller than the wavelength, the optical force along the propagation direction can

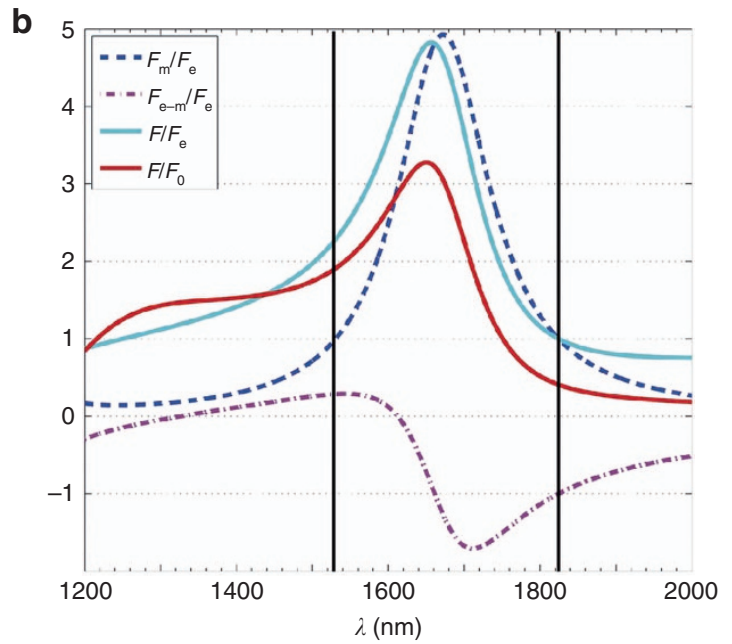

Figure 1 The contribution of electric and magnetic terms on optical force. (a) Real and imaginary parts of the electric and magnetic polarizabilities of a Si sphere with $a=230 \mathrm{~nm}$ normalized to $a^{3}$. (b) Electric, magnetic and electric-magnetic interference contributions to the optical force on the Si particle. $F_{0}=k a^{3} / 2$. The right and left vertical lines mark the wavelengths corresponding to the first and second Kerker conditions, respectively. Reprinted with permission from Ref. 67, (C) 2011 Optical Society of America. 
a

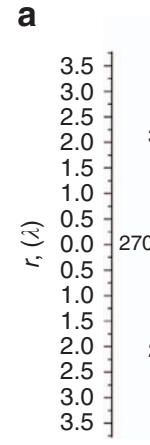

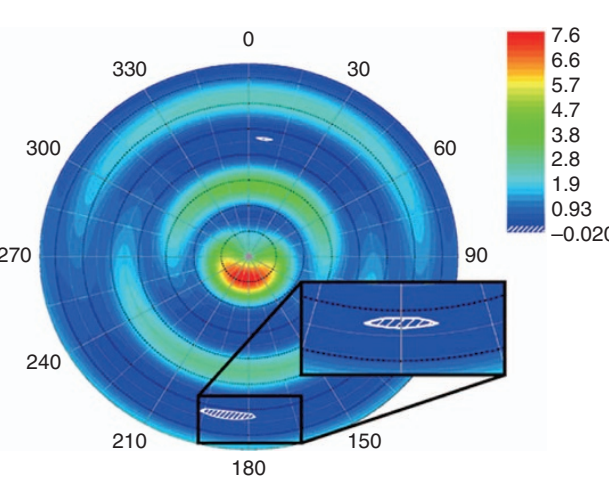

C

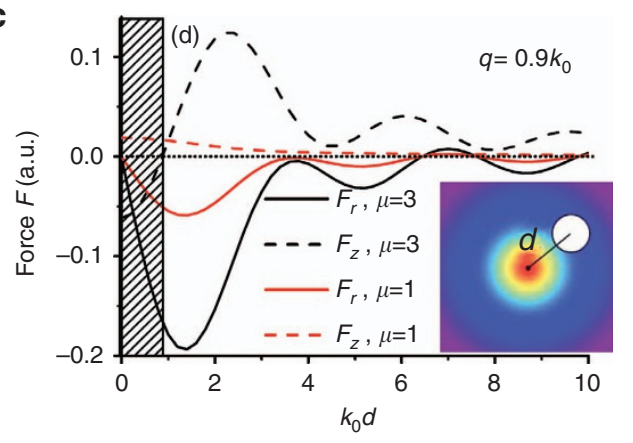

b

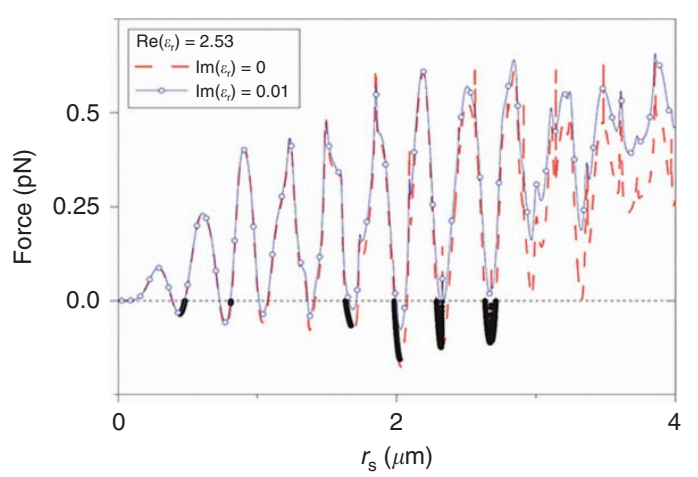

d

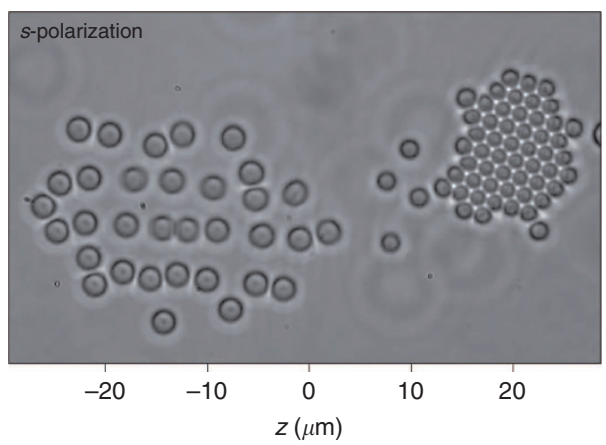

Figure 2 Optical trapping by structured beams. (a) Distribution of the optical force along the wave axis of a silver nanoparticle at $400 \mathrm{~nm}$. The inset shows the region of the NOF. (b) The optical force on a polystyrene sphere by a Bessel beam. Optical pulling force is possible for different radii of polystyrene particles. (c) The longitudinal forces $F_{z}$ and radial forces $F_{r}$ with a change in the radial distance between the particle and beam axis. The shaded region represents a stable pulling effect due to $F_{z}<0$ and $F_{r}<0$. Objects near the axis are confined by the gradient force and are transported in the propagation direction by the scattering force. (d) Polystyrene particles with radii of 800 and $1000 \mathrm{~nm}$ are separated by an s-polarized beam. Figure a reprinted with permission from Ref. 20, () 2010 Optical Society of America, b from Ref. 85, (c 2011 Nature Publishing Group, c from Ref. 22, ( 2011 American Physical Society, and d from Ref. 24, (c) 2013 Nature Publishing Group.

be expressed as ${ }^{18,22}$

$$
\begin{aligned}
\left\langle F_{z}\right\rangle= & \frac{\beta}{2}\left[\operatorname{Im}\left(\alpha_{\mathrm{e}}\right)\left|\boldsymbol{E}_{i}\right|^{2}+\operatorname{Im}\left(\alpha_{\mathrm{m}}\right)\left|\boldsymbol{H}_{i}\right|^{2}\right] \\
& -\frac{k^{4}}{12 \pi \varepsilon_{0} c} \operatorname{Re}\left(\alpha_{\mathrm{e}}\right) \operatorname{Re}\left(\alpha_{\mathrm{m}}\right) \operatorname{Re}\left(p_{z}\right)
\end{aligned}
$$

where $\beta$ is the longitudinal component of the wave vector, $\alpha_{\mathrm{e}, \mathrm{m}}$ is the electric (magnetic) polarizability and $p_{z}=\boldsymbol{e}_{z}\left(\boldsymbol{E}_{\mathrm{i}} \times \boldsymbol{H}_{\mathrm{i}}^{*}\right)$. From Equation (14), one can see that a negative force requires a small longitudinal wave vector and a large $p_{z}$ in the forward direction, that is, a small input photon momentum and a large output momentum in the propagation direction. The scattering along the propagation direction can be simultaneously maximized due to the interference of radiation multipoles. Then, the optical force can be pulling (see Figure $2 b$ ). The time-averaged optical force can be divided into the incident term and the interference term ${ }^{85}$. The large recoil force from the interference term may overcome the force from the incident terms, resulting in an attractive optical force. In addition, particles with material parameters close to the zero-backward condition $(\varepsilon=\mu)$ also tend to be pulled by light beams because under these conditions, forward scattering is maximal. However, stable trapping requires not only a negative force in the axis direction but also in the radial direction, that is, the optical force should be on the beam axis and point towards the light source ${ }^{22}$. In this way, a particle around the beam axis may be attracted to the beam center and be pulled to the light source in stable trajectories
(Figure 2c).For core-shell or hollow metallic particles, negative scattering force was found at Fano resonance, where the intensity of the forward scattering was much stronger than that of the backward scattering ${ }^{98}$. Hence, the enhanced forward scattering can give rise to a large recoil force. The incident and scattering light field can also be structured to obtain higher trapping stiffness. On the basis of the principle of maximal stiffness for a beam splitter, Taylor et al. ${ }^{99}$ demonstrated that structured scattering of particles improved the stiffness of optical trapping by $\sim 30$-fold. This approach could be a new way to perform OM.

Recently, experiments demonstrating tractor beams have been conducted. Lee et al. used an optical solenoid beam to trap and move a colloidal silica sphere against the direction of light propagation ${ }^{84}$. The solenoid beam exerted both intensity-gradient and phase-gradient forces on an object. By alternating the independent helical phase profile, the object could be transported downstream and retrograde upstream for a long distance. Similarly, the bidirectional transport of an optical conveyor was achieved by changing the relative phase of a Bessel beam ${ }^{21}$. This type of tractor beam has the potential to exert stable trapping with uniform speed, even on multiple objects. A tractor beam generated through the interference of a single Gaussian beam and its reflection also demonstrated the pulling effect ${ }^{24}$. Different sizes of particles can be sorted by switching between $s$ - and p-polarized beams (Figure 2d). Subsequently, polarization-controlled tractor beams have been demonstrated to stably transport spherical particles for over tens of centimeters in gaseous environments ${ }^{100}$. For absorbing 
particles in a gaseous medium, the gas-dynamic force (that is, photophoretic force) is dominant compared to the optical force ${ }^{101}$. By controlling the polarization of incident light (radially polarized or azimuthally polarized), the heat source distribution on an Au-coated hollow dielectric sphere can be significantly changed, thus determining the direction of the photophoretic force ${ }^{100}$.

In addition to the optical forces directly arising from lightmatter interactions, optical binding forces can emerge from the momentum exchange between multiple particles. Light-mediated optical binding offers a new method of self-assembly, organization and cell sorting. Moreover, the combination of scattering force and optical binding forces can produce stable optical trapping, even for a plane wave. Grzegorczyk et al. ${ }^{102}$ designed a trapping configuration with two series of fixed nanoparticles. The two walls of the nanoparticles interact with an incident plane wave and induce optical binding forces. By optimizing the locations of the fixed nanoparticles, they can create a potential well to stably trap one or more nanoparticles. Compared to microscale particles, stronger trapping forces are required to overcome the thermal forces for fine control of objects at the nanoscale. In the Rayleigh regime, both metallic and dielectric particles have similar scattering patterns ${ }^{103}$, so the gradient force is still a dominant factor for stable trapping of small particles in conventional optical tweezers. However, the optical binding forces of metallic nanoparticles have been found to be $>20$ times larger than the gradient force ${ }^{104}$, and they decayed slower than the gradient force ${ }^{105}$, which enables extremely stable nanometer manipulation. Hence, optical binding has potential applications in trapping small particles and assembling nanostructures. The spheroidal nanoparticles could arrange themselves into clusters, chains, photonic lattices and linear lines by optical binding, as shown in Figure $3 \mathrm{a}$ and $3 \mathrm{~b}$. Alternatively, computer-generated holographic tweezers can also be used to organize particles into such nanostructures in three dimensions (Figure $3 \mathrm{c}$ and $3 \mathrm{~d}$ ).

Because light carries not only linear momentum but also angular momentum (AM), the AM may be transported in optical tweezers to rotate particles in different ways (Figure 4a). In general, the torque from the transport of the linear momentum of incident waves is conservative and transient, whereas the torque due to the transport of $\mathrm{AM}$ is non-conservative and can continuously rotate particles with absorption, birefringence or asymmetry ${ }^{113-116}$. As discussed in 'Fundamentals of optical forces and computational approaches', the AM of light has two forms: spin angular momentum (SAM) and orbital angular momentum (OAM). Both SAM and OAM can be conveyed to the particle through absorption and scattering ${ }^{117}$ and result in torque that rotates the object ${ }^{118-124}$. The mechanical effect of AM has transformed optical tweezers into optical spanners ${ }^{125}$, attracting attention for their various potential applications. Apart from the SAM and OAM of light, optical absorption is essential for a homogeneous isotropic spherical particle to experience torque. A photon can transfer both forms of AM to the absorbing particle. Using a custom magneto-optic manipulator, the torque on absorbing microspheres caused by the transfer of SAM is directly measured ${ }^{126}$. This torque can be used in OM, for example, ultrafast spinning at the frequency of several $\mathrm{kHz}$ of the absorbing gold nanoparticles irradiated by circularly polarized light has been achieved ${ }^{127}$. Rubinsztein-Dunlop and colleagues experimentally observed the rotation of absorptive particles due to the absorption of OAM from a singularity beam ${ }^{128}$. a

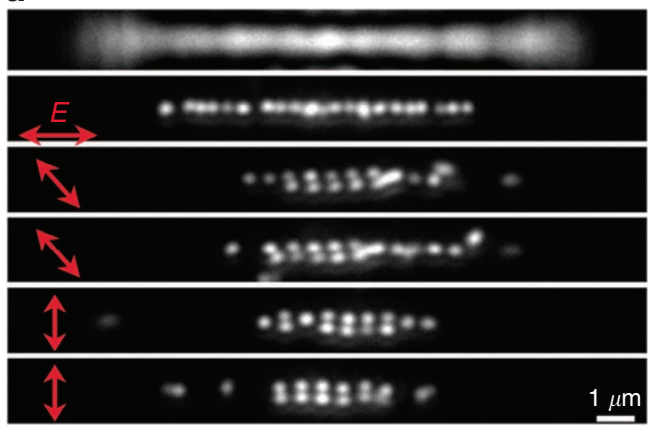

C

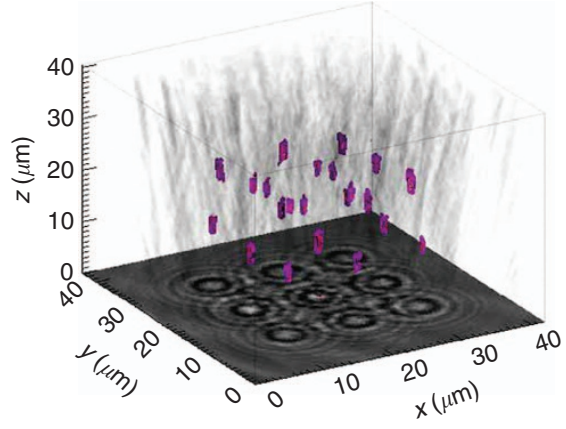

b

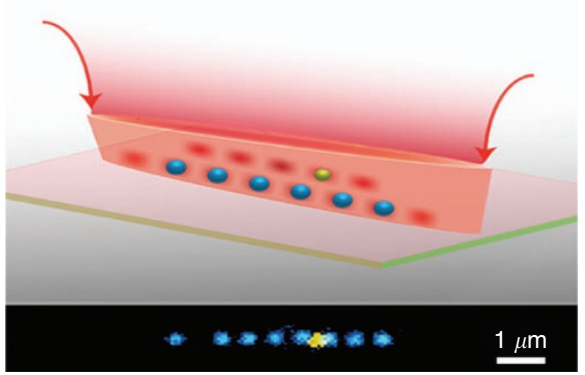

d
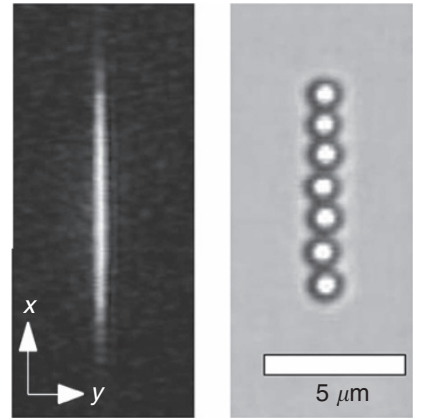

Figure 3 Optical binding forces between multiple particles. (a) Induced by optical binding, Ag nanoparticles with a $50 \mathrm{~nm}$ radius assemble into lines for different polarization directions. (b) Ag nanoparticles are formed into linear chains under coherent light. (c) Twenty-two silica spheres are arranged into a crystalline lattice with holographic optical tweezers. The colored regions are the positions of the particles in the lattice. (d) Holographic optical line tweezers in the focal plane and the image of seven silica spheres trapped along the line. Figure a reprinted with permission from Ref. 106 and $\mathbf{b}$ from Ref. 107 , (C) 2013 American Chemical Society, c from Ref. 89, (c) 2011 Optical Society of America, and d from Ref. 108, () 2008 American Physical Society. 
a

C
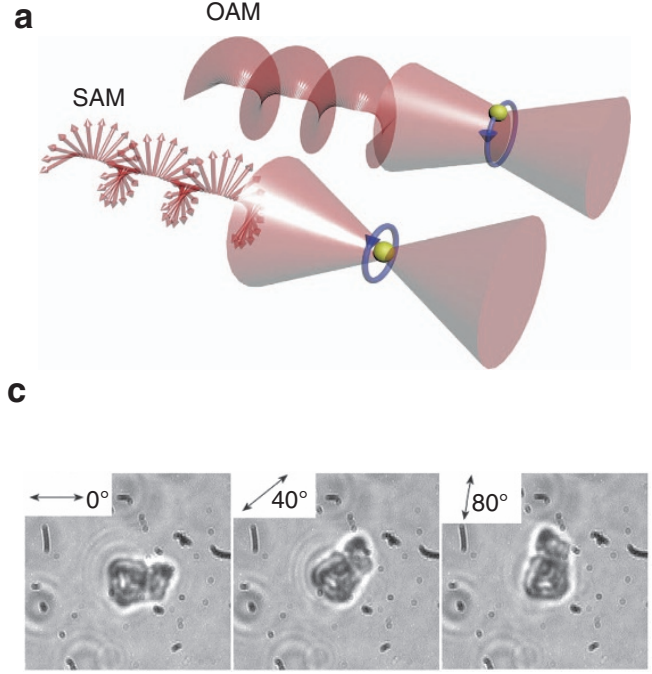

b

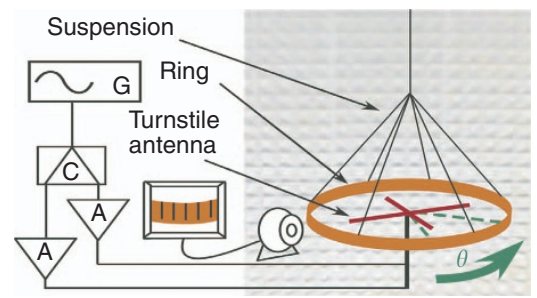

d

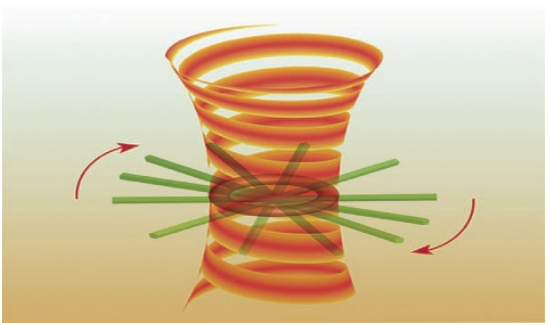

Figure 4 Optical torque via angular momentum. (a) In optical trapping, the transfer of OAM (associated with helical phase fronts) rotates a particle around the beam axis, whereas the transfer of SAM (associated with a polarization vector) causes a particle to spin around its own center. (b) Experimental set-up of the transfer of OAM to a suspended copper ring in the microwave region. (c) The trapped birefringent particles were rotated by rotating the half-wave plate that controlled the polarization of the trapping beam. (d) The rotation of a single silver nanowire was due to plasmonic interactions of nanowires with the optical vortex beam. Figure b reprinted with permission from Ref. 109, @ 2014 American Physical Society; c from Ref. 110 and its erratum 111 , @ 1998 Nature Publishing Group, and d from Ref. 112, (C) 2013 American Chemical Society.

The absorbing particle experienced optical torque along the beam axis on which the incident AM was defined. The rotation direction reversed when the sign of the incident AM was changed ${ }^{129}$. However, absorption may cause unwanted thermal effects, obstructing stable particle trapping.

To create optical torque predominantly from scattering, the scattered light should be made to exhibit a twist in the azimuthal direction, which means the AM carried by the scattered beam should be different from that of the incident beam. The scattering-dominant optical torque can be enhanced by using multipolar plasmon resonance. The AM transfer between photons and flat symmetrybreaking nanostructures was theoretically studied, and it was shown that resonant scattering can contribute up to $80 \%$ of the optical torque $^{117}$. The scattering-dominant optical torque makes it possible to manipulate microscale dielectric particles that have negligible absorption. Emile et al. ${ }^{109}$ used a torsion pendulum to demonstrate the transfer of OAM carried by an electromagnetic wave, which was generated by a pair of turnstile antennas, to a suspended copper ring in the microwave region. A negative torque (NOT) occurs due to AM conservation when the AM of the scattered light in the $z$-component is larger than that of the incident light (Figure $4 \mathrm{~b}$ ).

In addition, the conservation laws for spin and orbital angular momenta scattered by a small particle help us to understand the underlying physical nature of $\mathrm{NOT}^{130,131}$. Conversion can occur in optically inhomogeneous anisotropic media, and the transformation of SAM to OAM may give rise to 'left-handed' torque ${ }^{132-134}$. An inhomogeneous and anisotropic macroscopic object (form-birefringent nanostructured glass plates) was recently used to demonstrate the left-handed mechanical effect, which rotates the object in the opposite direction of the incident $\mathrm{AM}^{134}$. As it is not easy to directly observe and rotate a macroscopic object, D Hakobyan combined the rotation with the rotational Doppler effect and identified the rotation direction of the macroscopic object using the spatiotemporal interferograms of the transmission beam with a reference beam. This counterintuitive mechanical effect is analogous to the abovementioned 'tractor beams' and may provide insights into the mechanism of the spin-orbit interaction.

There are several other ways to rotate objects, such as using birefringent particles, chiral structures and metallic nanowires ${ }^{110,112,135}$, as shown in Figure $4 \mathrm{c}$ and $4 \mathrm{~d}$. For stable rotation of metallic nanowires using the optical vortex shown in Figure $4 \mathrm{~d}$, the length of the metallic nanowires must be longer than the bright ring of the optical vortex. Higher topological charges of the optical vortex lead to larger bright rings ${ }^{112}$; hence, shorter nanowires cannot be rotated in the dark core of the optical vortex. More generally, an unsymmetric object, such as a nanorod or an oblate spheroid ${ }^{136-138}$, may experience torque (in addition to an optical force) when illuminated by a Gaussian beam (without AM) because the force distribution inside the object is inhomogeneous. Certainly, the torque will vanish when the equilibrium orientation and position are reached. The torque can be observed in micrometer-scale structures immersed in water and under the illumination of laser light ${ }^{139}$. This achievement was a big step towards the OM of microscopic objects.

\section{FORCE ENHANCEMENT BY PLASMONICS AT THE NANOSCALE}

Although conventional optical tweezers, based on focused propagating beams, have been extensively employed to manipulate micrometer objects, it is difficult to stably and accurately trap particles with radii much smaller than the wavelength. The precise manipulation of small particles at the nanoscale is crucial in biosciences and in atomic and material physics for handling living viruses ${ }^{140-142}$, cooling neutral atoms ${ }^{143-145}$ and fabricating nanoscale structures ${ }^{146}$. Hence, it is crucial to enhance the trapping forces for small particles. The main problem for trapping nanoparticles is that the gradient force decreases rapidly with decreasing particle size (the force is proportional to the third power of the particle radius). Small particles may easily escape the trapping potential well due to Brownian motion. Meanwhile, the diffraction limit of the trapping beam also hinders accurate trap confinement. Simply using higher numerical aperture lenses or increasing the laser power does not significantly improve the optical 
tweezer performance. In addition, high-power lasers cause instant damage to the particles due to thermal effects.

Fortunately, plasmon nanotweezers based on surface plasmon offer an alternative approach to scale the trapped objects down to the nanoscale. Surface plasmons are collective oscillations of charges at flat metal-dielectric interfaces ${ }^{147}$ (surface plasmon polaritons, SPPs) or bound electron plasmas on particles ${ }^{43}$ (localized surface plasmons, LSPs). The excited SPPs can be used to focus light energy far beyond the diffraction limit, which can greatly increase the precision of OM. Another merit of SPPs is that the evanescent field decays rapidly from the interface, inducing strong gradient forces and making the trapping more stable. Because evanescent wave trapping in the near-field is not subject to the diffraction limit and does not require a high numerical aperture lens, it is an attractive alternative method to manipulate nanoscale objects.

As the concept of using SPPs to enhance the trapping force with evanescent fields was introduced for optical trapping, various configurations have been proposed, such as channeled waveguide ${ }^{148,149}$, external laser-illuminated metal tips and metallic layers with a subwavelength aperture ${ }^{150}$. These configurations can trap and move particles at the nanoscale. The local electric field is highly enhanced so that the induced optical forces are much larger than the forces from thermal fluctuations or gravity. For example, Zhang and colleagues ${ }^{151}$ used a metallic substrate coupled with a dielectric waveguide to confine a hybrid plasmonic mode between the waveguide and the substrate. The magnitude of optical force was greatly increased compared to the force when using a dielectric substrate. Subsequently, experimental realizations of SPP trapping were demonstrated using metal-dielectric interfaces, whereby surface plasmons are confined to subwavelength scales. Volpe et al. ${ }^{152}$ first observed and measured the plasmon radiation forces on dielectric spheres. They used a linearly polarized beam to impinge upon the metal-water interface through a prism to obtain surface plasmons. A polystyrene bead sample was placed between two separated cover slips. A photonic force microscope, which could obtain the optical force by analyzing the particle Brownian motion, was used to trap the probe particle and detect its position. To compare the forces in the surface plasmon resonant case and nonresonant case, the polarization of the incident electromagnetic wave can be changed to couple (transverse magnetic polarized) or decouple (transverse electric polarized) surface plasmons with the dielectric particle. In the experiment, the optical force magnitude at surface plasmon resonance was $\sim 40$ times larger than that off resonance. Wane et al. ${ }^{152,153}$ placed gold particles very close $(5 \mathrm{~nm})$ to the gold film so that the particles could strongly couple with the gold film (Figure 5a). The momentum from the photons was effectively transferred to the nanoparticles and greatly reduced the illumination power. Exploiting surface plasmons not only improves the trapping well of optical tweezers but also makes parallel trapping of specific particles possible. Righini et al. ${ }^{154,155}$ replaced the flat gold film with patterned gold disks in the Kretschmann configuration (Figure 5b), which could confine local fields to trap particles at desired positions. This configuration has potential applications in on-chip devices with non-focused beams and low laser power.

a

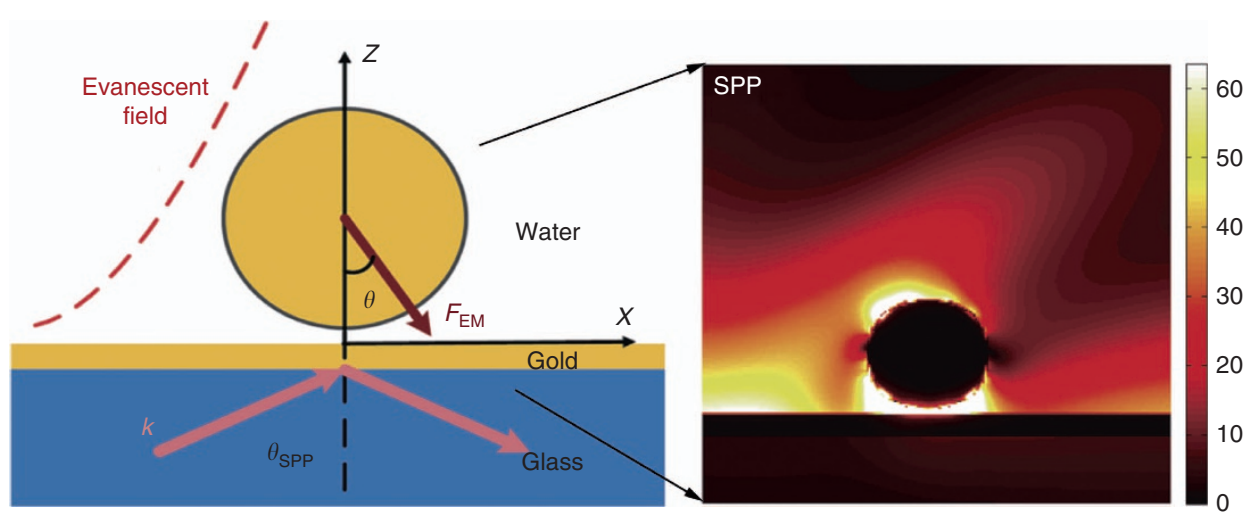

b

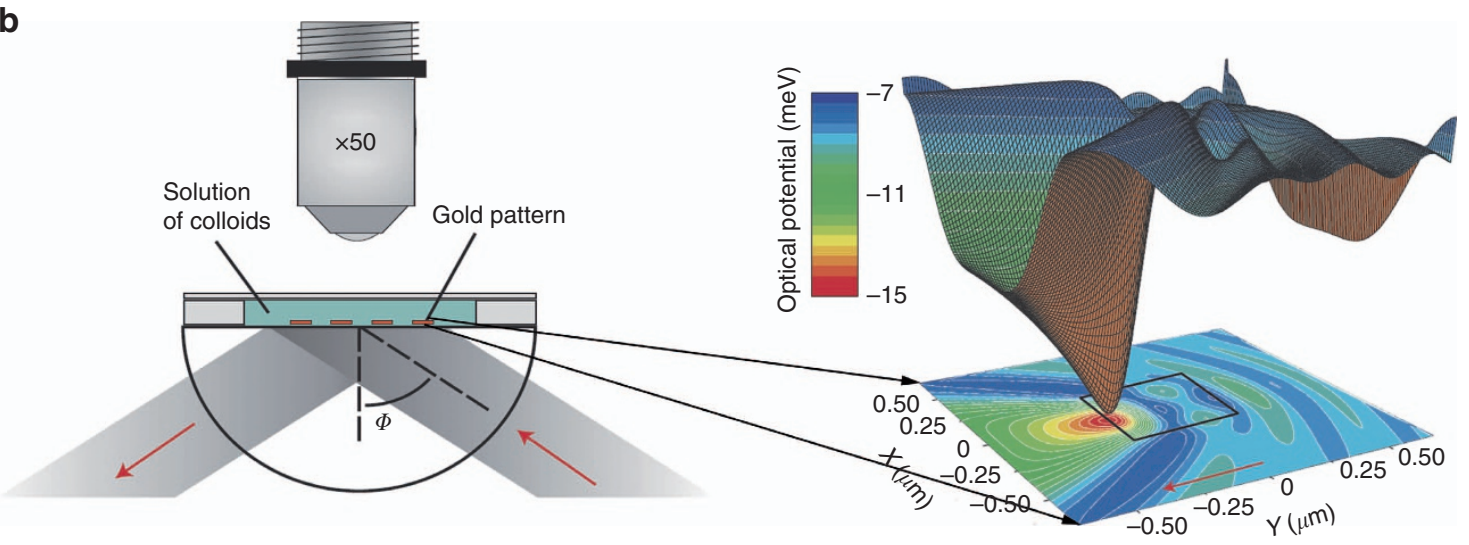

Figure 5 The enhancement of optical force via plasmonics. (a) Schematic of OM and power flow magnitudes for SPP excitation. (b) Scheme of the experimental configuration and the computed optical potential for a $200 \mathrm{~nm}$ polystyrene bead near a gold pad. Figure a reprinted with permission from Ref. 153, () 2009 American Chemical Society, and b from Ref. 154, ( 2007 Nature Publishing Group. 
Recently, various new plasmonic structures, which are tunable, more efficient and generate less heat, have been proposed to dynamically trap objects ${ }^{156-160}$. Sainidou et al. ${ }^{157}$ theoretically designed open microcavities to trap and control nanoparticles by tuning the wavelength of applied light ${ }^{156}$. The position of nanoparticles inside the nanocavities can significantly vary the resonance modes of the plasmonic structure, producing a series of equilibrium positions for particular illumination wavelengths. Thus, the incident light can precisely manipulate the trapped nanoparticles. Similarly, by exploiting the strong effect of trapped particles on the local field, a self-induced back-action (SIBA) plasmonic trap was devised to dynamically and automatically control objects within the nanoaperture of a metallic film (Figure 6a). When the object moves away from the equilibrium point, a restoring force pulls it back because the movement of the object decreases the optical transmission through the aperture, leading to a backward force to compensate the decrement of photon momentum rate. Meanwhile, the SIBA approach greatly reduced the required optical intensity for trapping, improving the trapping efficiency. A subsequent experiment ${ }^{162}$ revealed the optomechanical nature of the SIBA effects to provide optimum conditions for SIBAbased tweezers. More recently, plasmonic structures have been integrated with electric and thermal fields to trap and lock nanoparticles with high resolution and lower input power (Figure $6 \mathrm{~b})^{161}$. In addition, exciting LSP is a good approach to trap nanoscale objects by coupling directly with the incident electromagnetic wave. A strong light spot can be generated between the gaps of metallic nanostructures, thus creating a giant optical trapping force. Various plasmonic dimers, such as dipole antennas, bowtie nanoantenna arrays and double-nanohole setups ${ }^{158,160,163,164}$, have been widely used to control nanoparticles. LSP can also be applied to on-chip devices to simulatenously manipulate nanoscale beads or biological samples, such as living bacteria or cells, and may be extended to the molecule level to trap or sort single protein and DNA molecules.

\section{APPLICATIONS IN BIOCHEMICAL MANIPULATION}

Currently, optical traps are one of the most preferred methods for manipulating objects in microscopic systems in the fields of physics, chemistry, biology and engineering. In particular, optical tweezers with gradient forces have proven to be an ideal tool in single-molecule biophysics. With the aid of modern positional analysis apparatuses, the motion and forces of a trapped biomolecule during biochemical interactions can be measured and studied. In the past two decades, a variety of biological mechanisms have been investigated using optical tweezers, such as the dynamics of motor molecules ${ }^{165}$, the motion of RNA polymerase during transcription (Figure 7a), the motion of ribosomes during translation ${ }^{170}$, protein folding ${ }^{171}$ and DNA-protein binding. Optical tweezers can also be used to trap and study single cells and organelles within cells ${ }^{172-174}$. However, fixed optical tweezers have some restrictions: they can only apply limited forces of $0.1-100 \mathrm{pN}$ and can measure a range of motion of $\sim 400 \mathrm{~nm}$ or less ${ }^{175}$. In this section, recent advances to overcome these restrictions and enable new manipulation features and biomedical applications are reviewed.

Recent studies of light-matter interactions have raised the upper limit of trapping forces by optimizing either the incident beam or the illuminated particles. Jannasch et al. ${ }^{176}$ demonstrated nanonewton optical trapping forces by applying an anti-reflection coating on highrefractive-index core-shell particles. These optimized particles can be
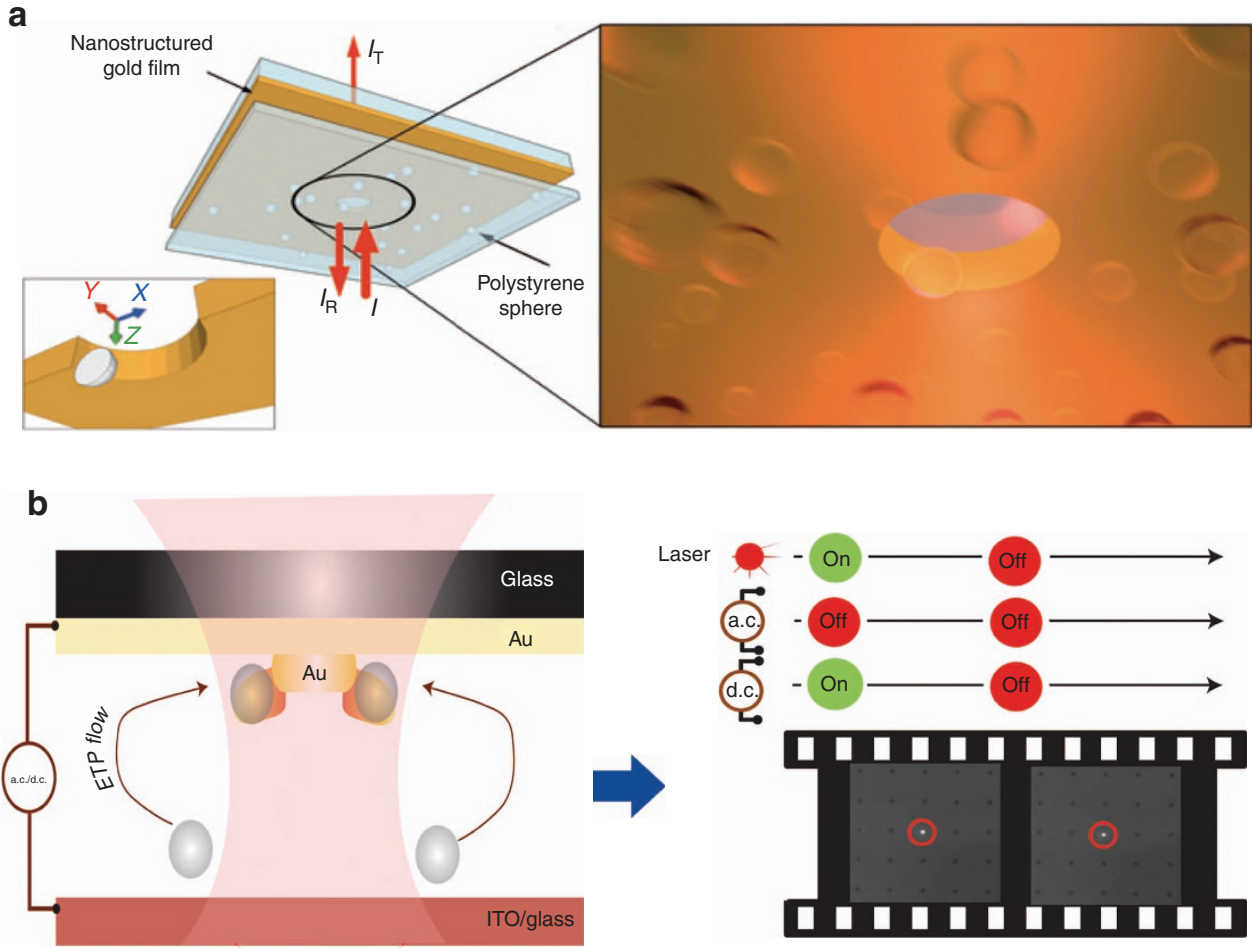

Figure 6 Plasmonic traps manipulating nanoparticles. (a) Self-induced back-action plasmonic trap for $100 \mathrm{~nm}$ polystyrene spheres in water. The trapped spheres play an active role: their positions strongly affect the aperture transmission, whereas departure from their equilibrium point leads to an automatic restoring force. Figure reprinted with permission from Ref. 156, @ 2009 Nature Publishing Group. (b) Experimental set-up for the electrothermoplasmonic nanotweezer (left). Particles are delivered to plasmonic hotspots and immobilized by an applied d.c. field (right). Figure reprinted with permission from Ref. 161, (C) 2015 Nature Publishing Group. 
a

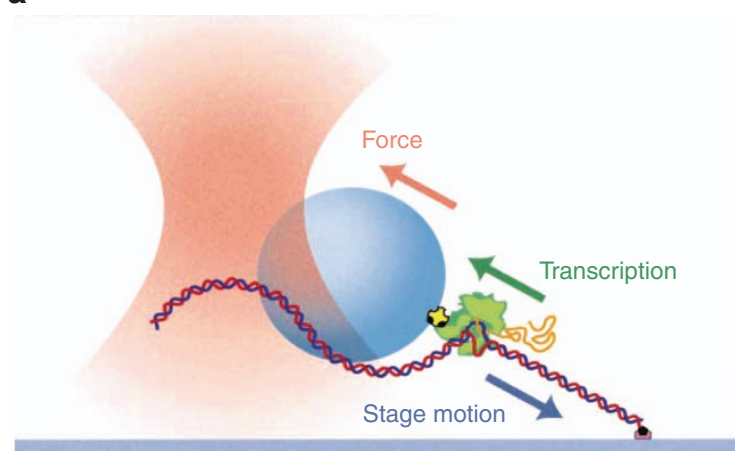

c

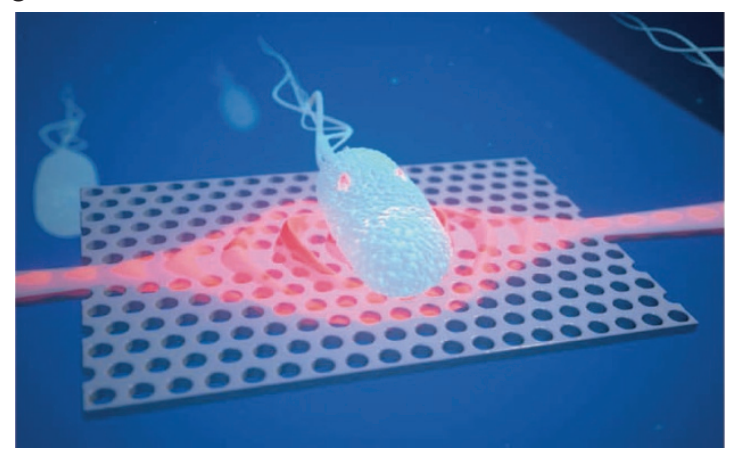

b

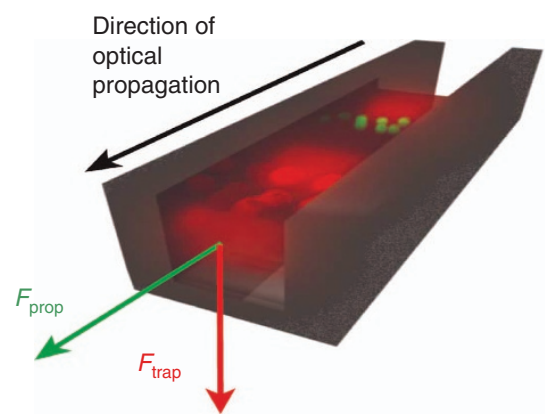

d

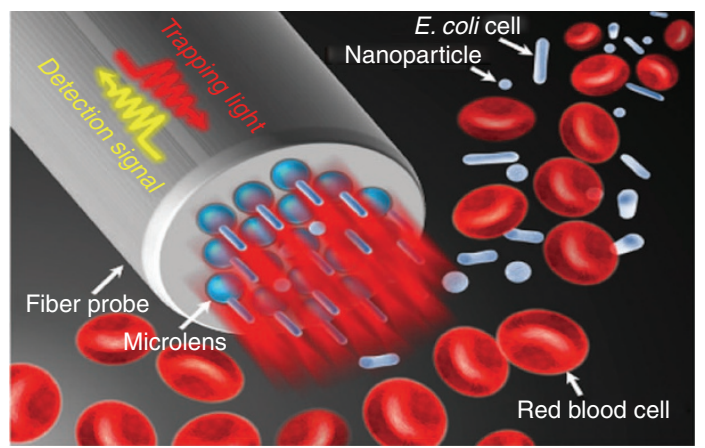

Figure 7 The applications of optical traps in biochemical manipulation. (a) Schematic of an optical tweezers-based assay for measuring the force on RNA polymerase during DNA transcription. (b) Schematic of the slot waveguide used to transport small particles and $\lambda$-DNA. (c) Artist's impression of a photonic crystal with a cavity for trapping bacteria. (d) Trapping and biosensing: parallel photonic nanojet array can be used to selectively trap and detect nanoparticles and biological cells. Figure a reprinted with permission from Ref. 166, () 2003 Elsevier, b from Ref. 167, () 2009 Nature Publishing Group, c from Ref. 168, () 2013 Royal Society of Chemistry (Great Britain), and d from Ref. 169, () 2016 American Chemical Society.

used as handles to exert force on biomolecules. In the nanonewton range, processes such as the unfolding of proteins and nucleic acids and intercellular interactions can be studied using optical tweezers. To overcome the difficulty of trapping subwavelength scale objects, a set of near-field methods have been developed based on SPP and waveguide structures ${ }^{167}$. The trapping and transportation of particles as small as $75 \mathrm{~nm}$, as well as $\lambda$-DNA molecules, were demonstrated using a slot waveguide (Figure $7 \mathrm{~b}$ ). Near-field trapping provides new abilities for manipulating small cells and biomolecules in space, which is a critical step in bioanalysis.

With the development of nanotechniques, the application of modern devices is not limited to OM. Micrototal analysis systems (lab-on-a-chip) can be easily combined with optical tweezers, making it possible to measure and explore the fluid-particle/ particle-particle interactions and stress fields around biological cells. The integrated 'lab-on-a-chip' platform may enable imaging, sensing, bioanalysis and diagnosis on a single chip ${ }^{177,178}$. On such a platform, the response of single cells to a range of gene manipulations or different biochemical environments may be recorded to study individual cellular functions ${ }^{179}$. Microfluidic systems consisting of a series of channels with diameters of $\sim 100 \mu \mathrm{m}$ are usually integrated on the platforms to assist the analysis. Therefore, OM over a relatively long distance can play an important role in these systems to controllably transport cells or biomolecules between areas. The distance of objects transported by fixed optical tweezers is greatly limited due to the short focal length of the lens, but this constraint can be broken by using 'nondiffraction' beams, such as Bessel ${ }^{21,93,180,181}$, Mathieu ${ }^{182,183}$ and
Airy beams ${ }^{97,184}$. Using this technique, an optical conveyor belt enabling bidirectional transportation was experimentally demonstrated. Again, particles optimized for optical pulling force can be used as handles attached to biomolecules. Integrating acoustooptic technology, which can generate arrays of such optical conveyors, will enable precise bidirectional transportation in three-dimensional space.

Near-field nanostructures can transport objects in a similar manner to 'non-diffraction' beams: the objects are trapped at the surface of the structure by gradient force and are continuously propelled by the scattering force. On the basis of current fabrication technology, these structures can be integrated with microfluidic systems on a 'lab-on-a-chip' platform to sort and fractionate biological molecules and cells ${ }^{185-187}$. A set of nanostructures has been developed to trap and transport biomolecules or cells, including waveguides and photonic crystals (Figure 7c). A structure with two micro-ring resonators with different resonant wavelengths integrated with a straight waveguide can be used to trap, transport and store particles ${ }^{188}$. By tuning the incident wavelength, particles can be transported and stored at different regions along the waveguide for a relatively long time. This device can be used to study cells' responses to a series of environments in an orderly manner. In another resonator-waveguide system ${ }^{189}$, the optical pulling force on a micro-ring resonator was achieved using the net scattering momentum resulting from the mode conversion in the resonator. Combined with the lateral optical equilibrium effect, a bidirectional optical conveyor was proposed such that trapped objects and the resonator itself could be transported 
a
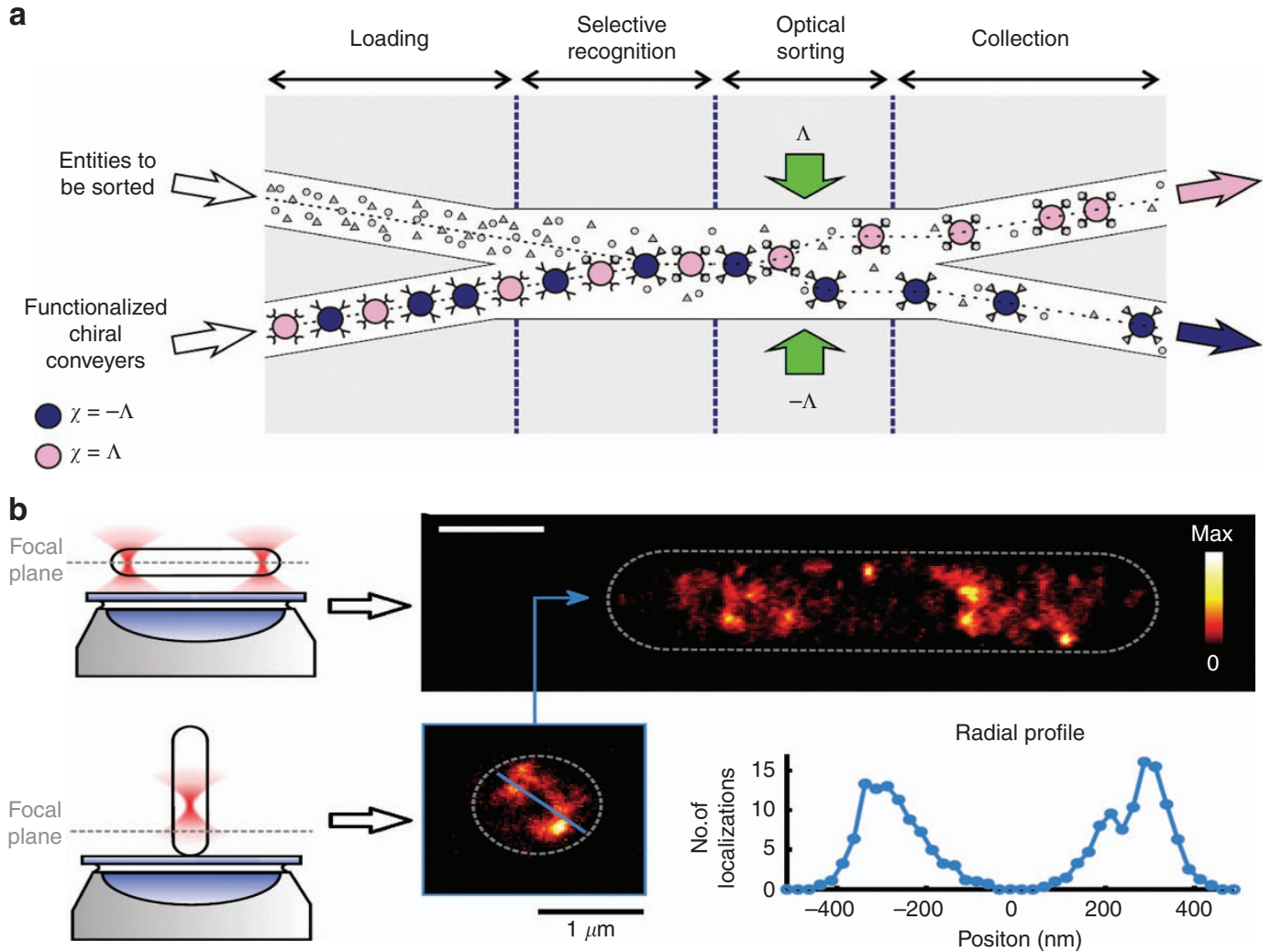

Figure 8 Optical sorting and imaging. (a) Optical chiral sorting for a random sequence of mixed chiral microparticles. (b) Holographic optical tweezers render high-resolution images of one cell from horizontal alignment and vertical alignment. Figure a reprinted with permission from Ref. 193, () 2014 Nature Publishing Group, and b from Ref. 194, (c) 2016 Nature Publishing Group.

simultaneously. The ring resonator can be used as a carrier to transport biomolecules and cells in two directions. More recently, scientists have developed additional functions for optical tweezers, such as biosensing (Figure $7 \mathrm{~d}$ ). Photonic nanojets are able to confine subwavelength light fields and detect backscasttering signals from trapped nanoparticles at low optical power ${ }^{169}$.

In addition to force and displacement, torque generation is common in diverse cellular processes, such as DNA packing, transcription, replication and the functioning of rotary biomotors. An optical torque wrench ${ }^{190}$ can generate torque on trapped objects by transferring the SAM of the incident light. In the set-up, a birefringent object, such as a nanofabricated quartz cylinder, was used as a handle with one end attached to the molecule of interest. Torque along the long axis of the handle was generated by rotation of the polarization of the trapping laser ${ }^{191}$. The torque generated by the biomolecule can be transferred to the handle and measured by detecting the change in polarization between the light entering and leaving the handle. This method has been used to detect the torque of DNA during supercoiling ${ }^{191}$, and that of RNA polymerase during transcription ${ }^{192}$. In addition, light beams carry not only linear momentum but also $\mathrm{AM}$, which can be applied to rotate particles in optical tweezers by transporting AM. By using a pre-designed optical set-up, left-handed or right-handed DNA/RNA-like molecules can be sorted if they are rotated in different directions, making it is possible to sort and manipulate individual DNA molecules (Figure 8a). Holographic optical tweezers can control the orientation of a cell at will and can achieve super-resolution using localization microscopy, yielding multiple perspectives of one sample with nanoscale localization precision
(Figure 8b) ${ }^{194}$. The new developments will lead to another revolution in $\mathrm{OM}$ and create abundant opportunities in bioscience ${ }^{195}$.

\section{CONCLUSIONS AND FUTURE PROSPECTS}

As a rapidly developing research field, there will be many possible trends and topics related to OM in future research. We have reviewed the new developments that have flourished in recent years. This area will continue to rapidly progress in the future.

On one hand, OM in inhomogeneous or complex backgrounds will attract increasing attention. Most manipulations are currently performed in water, air or another homogenous environment, such as a birefringent medium ${ }^{196}$. When the background is inhomogeneous, however, its scattering adds to that from the object and greatly contributes to the mechanical action on the body. For example, it has been shown that a simple dielectric interface can be used to achieve an optical tractor beam to transport oil droplets backward over long distances using only one beam ${ }^{23}$. In this situation, one of the most basic problems of $\mathrm{OM}$ is the optical force calculation, which stems from the momentum exchange between light and matter. For configurations with embedding backgrounds that are more complex than a uniform medium bounded only by a planar interface, interesting optical trapping and manipulation techniques, together with sophisticated calculations of the optical force in such situations, may attract growing interest.

On the other hand, the focus will be on the manipulation, sorting and separation of nanoscale objects with dimensions of tens of nanometers. The gradient force $F_{\mathrm{g}}$ of optical tweezers rapidly scales down as the linear dimensions $r$ of the object decrease, namely, $F_{\mathrm{g}} \sim r^{3}$. Therefore, objects with a typical size of tens of nanometers are 
challenging to trap and manipulate through gradient forces, as these forces are miniscule, and their potential is overcome by convection currents in the environment or by Brownian motion of such tiny bodies. Trapping and spatially controlling such objects in an accurate way at the nanometer scale is a key issue to building new functional nano-devices.

With the recent abundant advances, we anticipate the following research trends in OM. First, more novel beams will emerge as tractor beams by carefully constructing the properties of the electromagnetic field. The limitations of the current tractor beams will be overcome by increasingly powerful modern optics. Meanwhile, proper background materials, such as metamaterials, will also play an important role in optical trapping. New optical phenomena may help to reveal lightmatter interactions. Second, ultra-accurate manipulation requires finer structures, even down to the subnanoscale, at which quantum tunneling, nonlocal and nonlinear effects should be taken into account. Furthermore, the increasing miniaturization of nanotweezers makes it easier to implement them into integrated analytical platforms ${ }^{43}$. Multi-function sensors with high spatial resolution down to the microscale may be used to monitor various physical parameters, such as electric fields, magnetic fields, temperature, vibration and even radiation levels, by detecting the tweezered particles or the transmitted signal in a photonic crystal fiber ${ }^{197}$. With the development of nanotechnology, the size of these sensors can be soon reduced to nanometers. Meanwhile, trapping scale will be further narrowed to $<10 \mathrm{~nm}$ when optical tweezers are integrated with novel techniques or configurations. For example, a sub-10 nm parallel electronic trap fabricated by atomic layer lithography was able to rapidly electronically trap nanoparticles between long electrodes with ultralow bias voltages ${ }^{198}$. A promising next generation of nanophotonic traps using silicon nitride waveguides can outperform conventional table-top laser trapping in most respect, such as subnanometer manipulation resolution, the volume and stiffness of trapping and the maneuverability of devices ${ }^{199}$. OM may progress towards becoming multifunctional, highly compact and ultra-accurate, with diverse promising applications in biosciences, chemistry and engineering.

\section{CONFLICT OF INTEREST}

The authors declare no conflict of interest.

\section{ACKNOWLEDGEMENTS}

CWQ acknowledges the financial support from the National University of Singapore (no. R-263-000-678-133). MN-V is supported by the Spanish MINECO grants FIS2012-36113-C03-03, FIS2014-55563-REDC and FIS201569295-C3-1-P. DLG acknowledges financial support from the National Natural Science Foundation of China (no. 11504252), the Natural Science Foundation for the Youth of Jiangsu Province (no. BK20150306), and the Natural Science Foundation for Colleges and Universities in Jiangsu Province of China (no. 15KJB140008).

1 Ashkin A, Dziedzic JM, Bjorkholm JE, Chu S. Observation of a single-beam gradient force optical trap for dielectric particles. Opt Lett 1986; 11: 288-290.

2 Ashkin A. Acceleration and trapping of particles by radiation pressure. Phys Rev Lett 1970; 24: 156-159.

3 Dholakia K, Zemánek P. Colloquium: gripped by light: optical binding. Rev Mod Phys 2010; 82: 1767-1791.

4 Burns MM, Fournier J-M, Golovchenko JA. Optical binding. Phys Rev Lett 1989; 63 : 1233-1236.

5 Chaumet PC, Nieto-Vesperinas M. Optical binding of particles with or without the presence of a flat dielectric surface. Phys Rev B 2001; 64: 035422.

6 Almaas E, Brevik I. Possible sorting mechanism for microparticles in an evanescent field. Phys Rev A 2013; 87: 063826.
7 Wang MM, Tu E, Raymond DE, Yang JM, Zhang $\mathrm{H}$ et al. Microfluidic sorting of mammalian cells by optical force switching. Nat Biotechnol 2005; 23: 83-87.

8 Moffitt JR, Chemla YR, Smith SB, Bustamante C. Recent advances in optical tweezers. Annu Rev Biochem 2008; 77: 205-228.

9 Chang DE, Thompson JD, Park H, Vuletić V, Zibrov AS et al. Trapping and manipulation of isolated atoms using nanoscale plasmonic structures. Phys Rev Lett 2009; 103: 123004.

10 Reiserer A, Nölleke C, Ritter S, Rempe G. Ground-state cooling of a single atom at the center of an optical cavity. Phys Rev Lett 2013; 110: 223003.

11 Fazal FM, Block SM. Optical tweezers study life under tension. Nat Photon 2011; 5: 318-321.

12 Li HT, Zhou DJ, Browne H, Klenerman D. Evidence for resonance optical trapping of individual fluorophore-labeled antibodies using single molecule fluorescence spectroscopy. J Am Chem Soc 2006; 128: 5711-5717.

13 Nieto-Vesperinas M, Chaumet PC, Rahmani A. Near-field photonic forces. Philos Trans A Math Phys Eng Sci 2004; 362: 719-737.

14 Nieto-Vesperinas M, Sáenz JJ, Gómez-Medina R, Chantada L. Optical forces on small magnetodielectric particles. Opt Express 2010; 18: 11428-11443.

15 Wang SB, Chan CT. Lateral optical force on chiral particles near a surface. Nat Commun 2014; 5: 3307.

16 Viravathana P. Marr DWM. Optical trapping of titania/silica core-shell colloidal particles. J Colloid Interface Sci 2000; 221: 301-307.

17 Li ZP, Zhang SP, Tong LM, Wang PJ, Dong B et al. Ultrasensitive size-selection of plasmonic nanoparticles by Fano interference optical force. ACS Nano 2014; 8: 701-708.

18 Novitsky A, Qiu C-W, Lavrinenko A. Material-independent and size-independent tractor beams for dipole objects. Phys Rev Lett 2012; 109: 023902.

19 Sukhov S, Dogariu A. Negative nonconservative forces: optical 'tractor beams' for arbitrary objects. Phys Rev Lett 2011; 107: 203602.

20 Sukhov S, Dogariu A. On the concept of 'tractor beams'. Opt Lett 2010; 35 : 3847-3849.

21 Ruffner DB, Grier DG. Optical conveyors: a class of active tractor beams. Phys Rev Lett 2012; 109: 163903.

22 Novitsky A, Qiu C-W, Wang HF. Single gradientless light beam drags particles as tractor beams. Phys Rev Lett 2011; 107: 203601.

23 Kajorndejnukul V, Ding WQ, Sukhov S, Qiu CW, Dogariu A. Linear momentum increase and negative optical forces at dielectric interface. Nat Photon 2013; 7: 787-790.

24 Brzobohatý 0 , Karásek V, Šiler M, Chvátal L, Čižmár $T$ et al. Experimental demonstration of optical transport, sorting and self-arrangement using a "tractor beam'. Nat Photon 2013; 7: 123-127.

25 Dogariu A, Sukhov S, Sáenz J. Optically induced 'negative forces'. Nat Photon 2013; 7: $24-27$.

26 Ginis V, Tassin P, Soukoulis CM, Veretennicoff I. Enhancing optical gradient forces with metamaterials. Phys Rev Lett 2013; 110: 057401.

27 Chan J, Mayer Alegre TP, Safavi-Naeini AH, Hill JT, Krause A et al. Laser cooling of a nanomechanical oscillator into its quantum ground state. Nature 2011; 478: 89-92.

28 Dalvit D, Milonni P, Roberts D, da Rosa F. Casimir Physics. Springer: Berlin Heidelberg. 2011.

29 Sukhov S, Douglass KM, Dogariu A. Dipole-dipole interaction in random electromagnetic fields. Opt Lett 2013; 38: 2385-2387.

30 Rodriguez AW, McCauley AP, Woolf D, Capasso F, Joannopoulos JD et al. Nontouching nanoparticle diclusters bound by repulsive and attractive Casimir forces. Phys Rev Lett 2010; 104: 160402

31 Munday JN, Capasso F, Parsegian VA. Measured long-range repulsive CasimirLifshitz forces. Nature 2009; 457: 170-173.

32 Munday JN, Capasso F. Repulsive casimir and van der Waals forces: from measurements to future technologies. Int J Mod Phys A 2010; 25: 2252-2259.

33 Auñón JM, Qiu CW, Nieto-Vesperinas M. Tailoring photonic forces on a magnetodielectric nanoparticle with a fluctuating optical source. Phys Rev A 2013; 88: 043817.

34 Neuman KC, Chadd EH, Liou GF, Bergman K, Block SM. Characterization of photodamage to Escherichia coli in optical traps. Biophys J 1999; 77: 2856-2863.

35 Righini M, Ghenuche P, Cherukulappurath S, Myroshnychenko V, de Abajo FJG et al. Nano-optical trapping of rayleigh particles and Escherichia colibacteria with resonant optical antennas. Nano Lett 2009; 9: 3387-3391.

36 Rasmussen MB, Oddershede LB, Siegumfeldt H. Optical tweezers cause physiological damage to Escherichia coli and Listeria bacteria. Appl Environ Microbiol 2008; 74: 2441-2446.

37 Quidant R, Girard C. Surface-plasmon-based optical manipulation. Laser Photon Rev 2008; 2: 47-57.

38 Woerdemann M, Alpmann C, Esseling M, Denz C. Advanced optical trapping by complex beam shaping. Laser Photon Rev 2013; 7: 839-854.

39 Maragò OM, Jones PH, Gucciardi PG, Volpe G, Ferrari AC. Optical trapping and manipulation of nanostructures. Nat Nanotechnol 2013; 8: 807-819.

40 Dholakia K. Čižmár T. Shaping the future of manipulation. Nat Photon 2011; 5: 335-342.

41 Grier DG. A revolution in optical manipulation. Nature 2003; 424: 810-816.

42 Padgett M, Bowman R. Tweezers with a twist. Nat Photon 2011; 5: 343-348.

43 Juan ML, Righini M, Quidant R. Plasmon nano-optical tweezers. Nat Photon 2011; 5: 349-356.

44 Ashkin A, Dziedzic JM. Radiation pressure on a free liquid surface. Phys Rev Lett 1973; 30: 139-142. 
45 Baxter C, Loudon R. Radiation pressure and the photon momentum in dielectrics. J Mod Opt 2010; 57: 830-842.

46 Griffiths DJ. Resource letter EM-1: Electromagnetic momentum. Am J Phys 2012; 80 7-18.

47 Kemp BA, Grzegorczyk TM, Kong JA. Optical momentum transfer to absorbing mie particles. Phys Rev Lett 2006; 97: 133902.

48 Barnett SM. Resolution of the abraham-minkowski dilemma. Phys Rev Lett 2010; 104: 070401.

49 Novotny L, Hecht B. Principles of Nano-Optics. 2nd edn. Cambridge, UK: Cambridge University Press; 2012.

50 Jackson JD. Classical Electrodynamics. 3rd edn. New York, USA: Wiley; 1998.

51 Minkowski H. Die Grundgleichungen für die elektromagnetischen Vorgänge in bewegten Körpern. Math Ann 1910; 68: 472-525.

52 Minkowski H. Die Grundgleichungen für die elektromagnetischen Vorgänge in bewegten Körpern. Nachr Ges Wiss Göttn Math Phys KI 1908; 53: 111.

53 Pfeifer RNC, Nieminen TA, Heckenberg NR, Rubinsztein-Dunlop H. Colloquium: momentum of an electromagnetic wave in dielectric media. Rev Mod Phys 2007; 79 1197-1216.

54 Milonni PW, Boyd RW. Momentum of light in a dielectric medium. Adv Opt Photon 2010; 2: 519-553.

55 Kemp BA. Resolution of the Abraham-Minkowski debate: implications for the electromagnetic wave theory of light in matter. J Appl Phys 2011; 109: 111101.

56 Abraham M. Zur Elektrodynamik bewegter Körper. Rend Circ Matem Palermo 1909; 28: 1 .

57 Abraham M. Sull'Elettrodinamica di minkowski. Rend Circ Matem Palermo 1910; 30. 33.

58 Brevik I, Ellingsen SÅ. Detection of the Abraham force with a succession of short optical pulses. Phys Rev A 2012; 86: 025801.

59 Rikken GLJA, van Tiggelen BA. Observation of the intrinsic abraham force in timevarying magnetic and electric fields. Phys Rev Lett 2012; 108: 230402

60 Rikken GLJA, van Tiggelen BA. Measurement of the Abraham force and its predicted QED corrections in crossed electric and magnetic fields. Phys Rev Lett 2011; 107: 170401.

61 Zhu TT, Mahdy MRC, Cao YY, Lv HY, Sun FK et al. Optical pulling using evanescent mode in sub-wavelength channels. Opt Express 2016; 24: 18436-18444.

62 Qiu C-W, Ding WQ, Mahdy MRC, Gao DL, Zhang TH et al. Photon momentum transfer in inhomogeneous dielectric mixtures and induced tractor beams. Light Sci Appl 2015; 4: e278.

63 Bethune-Waddell M, Chau KJ. Simulations of radiation pressure experiments narrow down the energy and momentum of light in matter. Rep Prog Phys 2015; 78: 122401.

64 Brevik I. Experiments in phenomenological electrodynamics and the electromagnetic energy-momentum tensor. Phys Rep 1979; 52: 133-201.

65 García-Cámara B, Gonzalez F, Moreno F, Gomez-Medina RSaenz JJ et al. On the optical response of nanoparticles: directionality effects and optical forces. In: Hashim A, editor. Smart Nanoparticles Technology. InTech: Vienna, Austria, 2012

66 Chaumet PC, Rahmani A. Electromagnetic force and torque on magnetic and negativeindex scatterers. Opt Express 2009; 17: 2224-2234.

67 Nieto-Vesperinas M, Gomez-Medina R, Saenz JJ. Angle-suppressed scattering and optical forces on submicrometer dielectric particles. J Opt Soc Am A Opt Image Sci Vis 2011; 28: 54-60.

68 Fu YH, Kuznetsov Al, Miroshnichenko AE, Yu YF, Luk'yanchuk B. Directional visible light scattering by silicon nanoparticles. Nat Commun 2013; 4: 1527.

69 Kerker M, Wang DS, Giles CL. Electromagnetic scattering by magnetic spheres. J Opt Soc Am A Opt Image Sci Vis 1983; 73: 765-767.

70 Filonov DS, Krasnok AE, Slobozhanyuk AP, Kapitanova PV, Nenasheva EA et al. Experimental verification of the concept of all-dielectric nanoantennas. Appl Phys Lett 2012; 100: 201113.

71 Geffrin JM, García-Cámara B, Gómez-Medina R, Albella P, Froufe-Pérez LS et al. Magnetic and electric coherence in forward- and back-scattered electromagnetic waves by a single dielectric subwavelength sphere. Nat Commun 2012; 3: 1171.

72 Arias-González JR, Nieto-Vesperinas M. Optical forces on small particles: attractive and repulsive nature and plasmon-resonance conditions. J Opt Soc Am A Opt Image Sci Vis 2003; 20: 1201-1209.

73 Albaladejo S, Marqués MI, Laroche M, Sáenz JJ. Scattering forces from the curl of the spin angular momentum of a light field. Phys Rev Lett 2009; 102: 113602.

74 Berry MV. Optical currents. J Opt A Pure App/ Opt 2009; 11: 094001.

75 Antognozzi M, Bermingham CR, Harniman RL, Simpson S, Senior J et al. Direct measurements of the extraordinary optical momentum and transverse spin-dependent force using a nano-cantilever. Nat Phys 2016; 12: 731-735.

76 Smith DR, Pendry JB, Wiltshire MCK. Metamaterials and negative refractive index. Science 2004; 305: 788-792.

77 García-Etxarri A, Gómez-Medina R, Froufe-Pérez LS, López C, Chantada L et al. Strong magnetic response of submicron silicon particles in the infrared. Opt Express 2011; 19: 4815-4826.

78 Rolly B, Geffrin JM, Abdeddaim R, Stout B, Bonod N. Controllable emission of a dipolar source coupled with a magneto-dielectric resonant subwavelength scatterer. Sci Rep 2013; 3: 3063.

79 Sáenz JJ. Optical forces: Laser tractor beams. Nat Photon 2011; 5: 514-515.

80 Cao T, Mao LB, Gao DL, Ding WQ, Qu C-W. Fano resonant $\mathrm{Ge}_{2} \mathrm{Sb}_{2} \mathrm{Te}_{5}$ nanoparticles realize switchable lateral optical force. Nanoscale 2016; 8: 5657-5666.
81 Webb KJ, Shivanand. Negative electromagnetic plane-wave force in gain media. Phys Rev E Stat Nonlin Soft Matter Phys 2011; 84: 057602.

82 Webb KJ, Shivanand. Electromagnetic plane-wave forces on homogeneous material. J Opt Soc Am B 2012; 29: 1904-1910.

$83 \mathrm{Ng}$ J, Lin ZF, Chan CT. Theory of optical trapping by an optical vortex beam. Phys Rev Lett 2010; 104: 103601.

84 Lee SH, Roichman Y, Grier DG. Optical solenoid beams. Opt Express 2010; 18 : 6988-6993.

85 Chen J, Ng J, Lin ZF, Chan CT. Optical pulling force. Nat Photon 2011; 5: 531-534.

86 Čižmár T, Kollárová V, Bouchal Z, Zemánek P. Sub-micron particle organization by self-imaging of non-diffracting beams. New J Phys 2006; 8: 43.

87 Leach J, Sinclair G, Jordan P, Courtial J, Padgett MJ et al. 3D manipulation of particles into crystal structures using holographic optical tweezers. Opt Express 2004; 12: $220-226$.

88 Melville H, Milne GF, Spalding GC, Sibbett W, Dholakia K et al. Optical trapping of three-dimensional structures using dynamic holograms. Opt Express 2003; 11: 3562-3567.

89 Cheong FC, Krishnatreya BJ, Grier DG. Strategies for three-dimensional particle tracking with holographic video microscopy. Opt Express 2010; 18: 13563-13573.

90 Grier DG, Roichman Y. Holographic optical trapping. Appl Opt 2006; 45: 880-887.

91 Roichman Y, Grier DG. Projecting extended optical traps with shape-phase holography. Opt Lett 2006; 31: 1675-1677.

92 Roichman Y, Cholis I, Grier DG. Volumetric imaging of holographic optical traps. Opt Express 2006; 14: 10907-10912.

93 Čižmár T, Garcés-Chávez V, Dholakia K, Zemánek P. Optical conveyor belt for delivery of submicron objects. Appl Phys Lett 2005; 86: 174101.

94 Durnin J, Miceli JJ, Eberly JH. Diffraction-free beams. Phys Rev Lett 1987; 58 : 1499-1501.

95 Garcés-Chávez V, McGloin D, Melville H, Sibbett W, Dholakia K. Simultaneous micromanipulation in multiple planes using a self-reconstructing light beam. Nature 2002; 419: 145-147.

96 Berry MV, Balazs NL. Nonspreading wave packets. Am J Phys 1979; 47: 264-267.

97 Baumgartl J, Mazilu M, Dholakia K. Optically mediated particle clearing using Airy wavepackets. Nat Photon 2008; 2: 675-678.

98 Chen HJ, Liu SY, Zi J, Lin ZF. Fano resonance-induced negative optical scattering force on plasmonic nanoparticles. ACS Nano 2015; 9: 1926-1935.

99 Taylor MA, Waleed M, Stilgoe AB, Rubinsztein-Dunlop H, Bowen WP. Enhanced optical trapping via structured scattering. Nat Photon 2015; 9: 669-673.

100 Shvedov V, Davoyan AR, Hnatovsky C, Engheta N, Krolikowski W. A long-range polarization-controlled optical tractor beam. Nat Photon 2014; 8: 846-850.

101 Phuoc TX. A comparative study of the photon pressure force, the photophoretic force, and the adhesion van der Waals force. Opt Commun 2005; 245: 27-35.

102 Grzegorczyk TM, Kemp BA, Kong JA. Stable optical trapping based on optical binding forces. Phys Rev Lett 2006; 96: 113903.

103 Svoboda K, Block SM. Optical trapping of metallic Rayleigh particles. Opt Lett 1994; 19: 930-932.

104 Demergis V, Florin EL. Ultrastrong optical binding of metallic nanoparticles. Nano Lett 2012; 12: 5756-5760.

105 Bradshaw DS, Andrews DL. Optically induced forces and torques: Interactions between nanoparticles in a laser beam. Phys Rev A 2005; 72: 033816.

106 Yan ZJ, Shah RA, Chado G, Gray SK, Pelton M et al. Guiding spatial arrangements of silver nanoparticles by optical binding interactions in shaped light fields. ACS Nano 2013; 7: 1790-1802.

107 Yan ZJ, Manna U, Qin W, Camire A, Guyot-Sionnest P et al. Hierarchical photonic synthesis of hybrid nanoparticle assemblies. J Phys Chem Lett 2013; 4: 2630-2636.

108 Polin M, Roichman Y, Grier DG. Autocalibrated colloidal interaction measurements with extended optical traps. Phys Rev E 2008; 77: 051401.

109 Emile O, Brousseau C, Emile J, Niemiec R, Madhjoubi K et al. Electromagnetically induced torque on a large ring in the microwave range. Phys Rev Lett 2014; 112 . 053902.

110 Friese MEJ, Nieminen TA, Heckenberg NR, Rubinsztein-Dunlop H. Optical alignment and spinning of laser-trapped microscopic particles. Nature 1998; 394: 348-350.

111 Friese MEJ, Nieminen TA, Heckenberg NR, Rubinsztein-Dunlop H. erratum:Optical alignment and spinning of laser-trapped microscopic particles. Nature 1998; 395: 621 .

112 Yan ZJ, Scherer NF. Optical vortex induced rotation of silver nanowires. J Phys Chem Lett 2013; 4: 2937-2942.

113 Miyakawa K, Adachi H, Inoue Y. Rotation of two-dimensional arrays of microparticles trapped by circularly polarized light. Appl Phys Lett 2004; 84: 5440-5442.

114 Haefner D, Sukhov S, Dogariu A. Conservative and nonconservative torques in optical binding. Phys Rev Lett 2009; 103: 173602.

115 Dienerowitz M, Mazilu M, Reece PJ, Krauss TF, Dholakia K. Optical vortex trap for resonant confinement of metal nanoparticles. Opt Express 2008; 16: 4991-4999.

116 Roichman Y, Sun B, Stolarski A, Grier DG. Influence of nonconservative optical forces on the dynamics of optically trapped colloidal spheres: the fountain of probability. Phys Rev Lett 2008; 101: 128301.

117 Lee YE, Fung KH, Jin DF, Fang NX. Optical torque from enhanced scattering by multipolar plasmonic resonance. Nanophotonics 2014; 3: 343-350. 
118 O'Neil AT, MacVicar I, Allen L, Padgett MJ. Intrinsic and extrinsic nature of the orbital angular momentum of a light beam. Phys Rev Lett 2002; 88: 053601.

119 Canaguier-Durand A, Cuche A, Genet C, Ebbesen TW. Force and torque on an electric dipole by spinning light fields. Phys Rev A 2013; 88: 033831.

120 Friese MEJ, Enger J, Rubinsztein-Dunlop H, Heckenberg NR. Optical angularmomentum transfer to trapped absorbing particles. Phys Rev A 1996; 54: 1593-1596.

121 Normanno D, Capitanio M, Pavone FS. Spin absorption, windmill, and magneto-optic effects in optical angular momentum transfer. Phys Rev A 2004; 70: 053829.

122 Bliokh KY, Kivshar YS, Nori F. Magnetoelectric effects in local light-matter interactions. Phys Rev Lett 2014; 113: 033601.

123 Curtis J, Grier D. Structure of optical vortices. Phys Rev Lett 2003; 90: 133901.

124 Garcés-Chávez V, McGloin D, Padgett MJ, Dultz W, Schmitzer H et al. Observation of the transfer of the local angular momentum density of a multiringed light beam to an optically trapped particle. Phys Rev Lett 2003; 91: 093602.

125 Simpson NB, Dholakia K, Allen L, Padgett MJ. Mechanical equivalence of spin and orbital angular momentum of light: an optical spanner. Opt Lett 1997; 22: 52-54.

126 Capitanio M, Normanno D, Pavone FS. High-precision measurements of light-induced torque on absorbing microspheres. Opt Lett 2004; 29: 2231-2233.

127 Lehmuskero A, Ogier R, Gschneidtner T, Johansson P, Käll M. Ultrafast spinning of gold nanoparticles in water using circularly polarized light. Nano Lett 2013; 13: 3129-3134.

$128 \mathrm{He} \mathrm{H}$, Friese ME, Heckenberg NR, Rubinsztein-Dunlop H. Direct observation of transfer of angular momentum to absorptive particles from a laser beam with a phase singularity. Phys Rev Lett 1995; 75: 826-829.

129 Chen J, Ng J, Ding K, Fung KH, Lin ZF et al. Negative optical torque. Sci Rep 2014; 4: 6386.

130 Nieto-Vesperinas M. Optical torque on small bi-isotropic particles. Opt Lett 2015; 40 3021-3024.

131 Nieto-Vesperinas M. Optical torque: electromagnetic spin and orbital-angularmomentum conservation laws and their significance. Phys Rev A 2015; 92: 043843.

132 Marrucci L, Manzo C, Paparo D. Optical spin-to-orbital angular momentum conversion in inhomogeneous anisotropic media. Phys Rev Lett 2006; 96: 163905.

133 Canaguier-Durand A, Genet C. Chiral route to pulling optical forces and left-handed optical torques. Phys Rev A 2015; 92: 043823 .

134 Hakobyan D, Brasselet E. Left-handed optical radiation torque. Nat Photon 2014; 8 : 610-614.

135 Irrera A, Magazzù A, Artoni P, Simpson SH, Hanna S et al. Photonic torque microscopy of the nonconservative force field for optically trapped silicon nanowires. Nano Lett 2016; 16: 4181-4188.

136 Liu M, Zentgraf T, Liu YM, Bartal G, Zhang X. Light-driven nanoscale plasmonic motors. Nat Nanotechnol 2010; 5: 570-573.

137 Cao YY, Song WH, Ding WQ, Sun FK, Zhu TT. Equilibrium orientations of oblate spheroidal particles in single tightly focused Gaussian beams. Opt Express 2014; 22: 18113-18118.

138 Cao YY, Stilgoe AB, Chen LX, Nieminen TA, Rubinsztein-Dunlop H. Equilibrium orientations and positions of non-spherical particles in optical traps. Opt Express 2012; 20: 12987-12996.

139 Swartzlander Jr GA, Peterson TJ, Artusio-Glimpse AB, Raisanen AD. Stable optical lift. Nat Photon 2011; 5: 48-51.

140 Ashkin A, Dziedzic JM. Optical trapping and manipulation of viruses and bacteria. Science 1987; 235: 1517-1520.

141 Ashkin A, Dziedzic JM, Yamane T. Optical trapping and manipulation of single cells using infrared laser beams. Nature 1987; 330: 769-771.

142 Liang H, Vu KT, Krishnan P, Trang TC, Shin D et al. Wavelength dependence of cell cloning efficiency after optical trapping. Biophys J 1996; 70: 1529-1533.

143 Ashkin A, Gordon JP. Cooling and trapping of atoms by resonance radiation pressure. Opt Lett 1979; 4: 161-163.

144 Hänsch TW, Schawlow AL. Cooling of gases by laser radiation. Opt Common 1975; 13: 68-69.

145 Kasevich M, Chu S. Laser cooling below a photon recoil with three-level atoms. Phys Rev Lett 1992; 69: 1741-1744.

146 Liang H, Wright WH, Cheng S, He W, Berns MW. Micromanipulation of chromosomes in PtK2 cells using laser microsurgery (optical scalpel) in combination with laserinduced optical force (optical tweezers). Exp Cell Res 1993; 204: 110-120.

147 Liu YM, Zhang X. Recent advances in transformation optics. Nanoscale 2012; 4: 5277-5292.

148 Kawata S, Tani T. Optically driven Mie particles in an evanescent field along a channeled waveguide. Opt Lett 1996; 21: 1768-1770.

149 Woolf D, Loncar M, Capasso F. The forces from coupled surface plasmon polaritons in planar waveguides. Opt Express 2009; 17: 19996-20011.

150 Okamoto K, Kawata S. Radiation force exerted on subwavelength particles near a nanoaperture. Phys Rev Lett 1999; 83: 4534-4537.

151 Yang XD, Liu YM, Oulton RF, Yin XB, Zhang X. Optical forces in hybrid plasmonic waveguides. Nano Lett 2011; 11: 321-328.

152 Volpe G, Quidant R, Badenes G, Petrov D. Surface plasmon radiation forces. Phys Rev Lett 2006; 96: 238101.

153 Wang K, Schonbrun E, Crozier KB. Propulsion of gold nanoparticles with surface plasmon polaritons: evidence of enhanced optical force from near-field coupling between gold particle and gold film. Nano Lett 2009; 9: 2623-2629.
154 Righini M, Zelenina AS, Girard C, Quidant R. Parallel and selective trapping in a patterned plasmonic landscape. Nat Phys 2007; 3: 477-480.

155 Righini M, Volpe G, Girard C, Petrov D, Quidant R. Surface plasmon optical tweezers: tunable optical manipulation in the femtonewton range. Phys Rev Lett 2008; 100: 186804.

156 Juan ML, Gordon R, Pang YJ, Eftekhari F, Quidant R. Self-induced back-action optical trapping of dielectric nanoparticles. Nat Phys 2009; 5: 915-919.

157 Sainidou R, García de Abajo FJ. Optically tunable surfaces with trapped particles in microcavities. Phys Rev Lett 2008; 101: 136802.

158 Pang YJ, Gordon R. Optical trapping of a single protein. Nano Lett 2012; 12 : 402-406.

159 Zhao Y, Saleh AAE, Dionne JA. Enantioselective optical trapping of chiral nanoparticles with plasmonic tweezers. ACS Photon 2016; 3: 304-309.

160 Jensen RA, Huang IC, Chen O, Choy JT, Bischof TS et al. Optical trapping and twophoton excitation of colloidal quantum dots using bowtie apertures. ACS Photon 2016; 3: 423-427.

161 Ndukaife JC, Kildishev AV, Nnanna AGA, Shalaev VM, Wereley ST et al. Long-range and rapid transport of individual nano-objects by a hybrid electrothermoplasmonic nanotweezer. Nat Nanotechnol 2016; 11: 53-59.

162 Mestres P, Berthelot J, Aćimović SS, Quidant R. Unraveling the optomechanical nature of plasmonic trapping. Light Sci Appl 2016; 5: e16092.

163 Zhang WH, Huang LN, Santschi C, Martin OJF. Trapping and sensing $10 \mathrm{~nm}$ metal nanoparticles using plasmonic dipole antennas. Nano Lett 2010; 10: 1006-1011.

164 Roxworthy BJ, Ko KD, Kumar A, Fung KH, Chow EKC et al. Application of plasmonic bowtie nanoantenna arrays for optical trapping, stacking, and sorting. Nano Lett 2012; 12: 796-801.

165 Clancy BE, Behnke-Parks WM, Andreasson JOL, Rosenfeld SS, Block SM. A universal pathway for kinesin stepping. Nat Struct Mol Biol 2011; 18: 1020-1027.

166 Neuman KC, Abbondanzieri EA, Landick R, Gelles J, Block SM. Ubiquitous transcriptional pausing is independent of RNA polymerase backtracking. Cell 2003; 115: 437-447.

167 Yang AHJ, Moore SD, Schmidt BS, Klug M, Lipson M et al. Optical manipulation of nanoparticles and biomolecules in sub-wavelength slot waveguides. Nature 2009; 457: 71-75.

168 van Leest T, Caro J. Cavity-enhanced optical trapping of bacteria using a silicon photonic crystal. Lab Chip 2013; 13: 4358-4365.

169 Li YC, Xin HB, Liu XS, Zhang Y, Lei HX et al. Trapping and detection of nanoparticles and cells using a parallel photonic nanojet array. ACS Nano 2016; 10: 5800-5808.

170 Wen JD, Lancaster L, Hodges C, Zeri AC, Yoshimura SH et al. Following translation by single ribosomes one codon at a time. Nature 2008; 452: 598-603.

171 Cecconi C, Shank EA, Bustamante C, Marqusee S. Direct observation of the threestate folding of a single protein molecule. Science 2005; 309: 2057-2060.

172 Arneborg N, Siegumfeldt H, Andersen GH, Nissen P, Daria VR et al. Interactive optical trapping shows that confinement is a determinant of growth in a mixed yeast culture. FEMS Microbiol Lett 2005; 245: 155-159.

173 Oddershede LB. Force probing of individual molecules inside the living cell is now a reality. Nat Chem Biol 2012; 8: 879-886.

174 Sitters G, Laurens N, de Rijk EJ, Kress H, Peterman EJG et al. Optical pushing: a tool for parallelized biomolecule manipulation. Biophys J 2016; 110: 44-50.

175 Neuman KC, Nagy A. Single-molecule force spectroscopy: optical tweezers, magnetic tweezers and atomic force microscopy. Nat Methods 2008; 5: 491-505.

176 Jannasch A, Demirörs AF, van Oostrum PDJ, van Blaaderen A, Schäffer E. Nanonewton optical force trap employing anti-reflection coated, high-refractive-index titania microspheres. Nat Photon 2012; 6: 469-473.

177 Psaltis D, Quake SR, Yang CH. Developing optofluidic technology through the fusion of microfluidics and optics. Nature 2006; 442: 381-386.

178 Burger R, Kurzbuch D, Gorkin R, Kijanka G, Glynn M et al. An integrated centrifugoopto-microfluidic platform for arraying, analysis, identification and manipulation of individual cells. Lab Chip 2015; 15: 378-381.

179 Ramser K, Hanstorp D. Optical manipulation for single-cell studies. J Biophotonics 2010; 3: 187-206.

180 Paterson L, Papagiakoumou E, Milne G, Garcés-Chávez V, Tatarkova SA et al. Lightinduced cell separation in a tailored optical landscape. App/ Phys Lett 2005; 87: 123901.

181 Rendall HA, Marchington RF, Praveen BB, Bergmann G, Arita Y et al. High-throughput optical injection of mammalian cells using a Bessel light beam. Lab Chip 2012; 12: 4816-4820.

182 Yan SH, Li MM, Yao BL, Yu XH, Lei M et al. Accelerating nondiffracting beams. Phys Lett A 2015; 379: 983-987.

183 Chafiq A, Belafhal A. Radiation pressure cross section exerted on homogenous dielectric spherical particle by zeroth order Mathieu beams. J Quant Spectrosc Radiat Transfer 2016; 179: 170-176.

184 Baumgartl J, Hannappel GM, Stevenson DJ, Day D, Gu M et al. Optical redistribution of microparticles and cells between microwells. Lab Chip 2009; 9: 1334-1336.

185 MacDonald MP, Spalding GC, Dholakia K. Microfluidic sorting in an optical lattice. Nature 2003; 426: 421-424.

$186 \mathrm{Kim} \mathrm{J}$, Shin JH. Stable, free-space optical trapping and manipulation of sub-micron particles in an integrated microfluidic chip. Sci Rep 2016; 6: 33842.

187 Decrop D, Brans T, Gijsenbergh P, Lu JD, Spasic D et al. Optical manipulation of single magnetic beads in a microwell array on a digital microfluidic chip. Anal Chem 2016; 88: 8596-8603. 
188 Lin SY, Crozier KB. Planar silicon microrings as wavelength-multiplexed optical traps for storing and sensing particles. Lab Chip 2011; 11: 4047-4051.

189 Intaraprasonk V, Fan SH. Optical pulling force and conveyor belt effect in resonatorwaveguide system. Opt Lett 2013; 38: 3264-3267.

190 La Porta A, Wang MD. Optical torque wrench: angular trapping, rotation, and torque detection of quartz microparticles. Phys Rev Lett 2004; 92: 190801.

191 Deufel C, Forth S, Simmons CR, Dejgosha S, Wang MD. Nanofabricated quartz cylinders for angular trapping: DNA supercoiling torque detection. Nat Methods 2007; 4: 223-225.

192 Ma J, Bai L, Wang MD. Transcription under torsion. Science 2013; 340: 1580-1583.

193 Tkachenko G, Brasselet E. Optofluidic sorting of material chirality by chiral light. Nat Commun 2014; 5: 3577.

194 Diekmann R, Wolfson DL, Spahn C, Heilemann M, Schuttpelz M et al. Nanoscopy of bacterial cells immobilized by holographic optical tweezers. Nat Commun 2016; 7: 13711.

195 Hayat A, Mueller JPB, Capasso F. Lateral chirality-sorting optical forces. Proc Nat/ Acad Sci USA 2015; 112: 13190-13194.

196 Forgács P, Lukács Á, Romańczukiewicz T. Plane waves as tractor beams. Phys Rev D 2013; 88: 125007 .
197 Bykov DS, Schmidt OA, Euser TG, Russell PSJ. Flying particle sensors in hollow-core photonic crystal fibre. Nat Photon 2015; 9: 461-465.

198 Barik A, Chen XS, Oh SH. Ultralow-power electronic trapping of nanoparticles with sub-10 nm gold nanogap electrodes. Nano Lett 2016; 16: 6317-6324.

199 Ye F, Badman RP, Inman JT, Soltani M, Killian JL et al. Biocompatible and high stiffness nanophotonic trap array for precise and versatile manipulation. Nano Lett 2016; 16: 6661-6667.

(c) (1)(2) This work is licensed under a Creative Commons AttributionBY NC SA NonCommercial-ShareAlike 4.0 International License. The images or other third party material in this article are included in the article's Creative Commons license, unless indicated otherwise in the credit line; if the material is not included under the Creative Commons license, users will need to obtain permission from the license holder to reproduce the material. To view a copy of this license, visit http:// creativecommons.org/licenses/by-nc-sa/4.0/

(C) The Author(s) 2017 